\title{
THE RIEMANN MAPPING THEOREM FOR SEMIANALYTIC DOMAINS AND O-MINIMALITY
}

\author{
T. KAISER
}

\begin{abstract}
We consider the Riemann Mapping Theorem in the case of a bounded simply connected and semianalytic domain. We show that the germ at 0 of the Riemann map (i.e. biholomorphic map) from the upper half plane to such a domain can be realized in a certain quasianalytic class if the angle of the boundary at the point to which 0 is mapped, is greater than 0 . This quasianalytic class was introduced and used by Ilyashenko in his work on Hilbert's $16^{\text {th }}$ problem. With this result we can prove that the Riemann map from a bounded simply connected semianalytic domain onto the unit ball is definable in an o-minimal structure, provided that at singular boundary points the angles of the boundary are irrational multiples of $\pi$.
\end{abstract}

\section{Introduction}

One of the central theorems in complex analysis is the

\section{Riemann Mapping Theorem}

Let $\Omega \varsubsetneqq \mathbb{C}$ be a simply connected domain in the plane. Then $\Omega$ can be mapped biholomorphically onto the unit ball $B(0,1)$.

A nice overview of its proofs and their history can be found in Remmert [29].

'Riemann maps' are in general transcendental and not algebraic functions. The goal of this paper is to show a 'tame' content of the Riemann Mapping Theorem. An important framework of 'tame' geometry is given by the category of semialgebraic sets and functions. Semialgebraic sets and functions have very nice finiteness properties (see Bochnak et al. [3]). A more general framework to study sets with singularities which still exhibit a nice behaviour is given by the category of subanalytic sets and functions (see Bierstone-Milman [2], Denef-Van den Dries [6], Lojasiewicz [24] and Shiota [31]). But we lose some of the finiteness properties we have in semialgebraic geometry. These are still valid, however, if we restrict ourselves to globally subanalytic sets and functions, i.e. to sets and functions which are subanalytic in the ambient projective space (compare with Van den Dries [7] and Van den Dries-Miller [10]). We lose little here since all bounded subanalytic sets are globally subanalytic.

2000 Mathematics Subject Classification 03C64, 32B20, 30C20, 30E15, 30D60, 30D05, $37 \mathrm{E} 35$.

Keywords and phrases: o-minimal structures, semianalytic sets, Riemann Mapping Theorem, quasianalytic classes, dynamical systems

Supported by DFG-project KN202/5-2 
The question is now the following:

Given a bounded simply connected domain in the plane which is semianalytic (in $\mathbb{R}^{2}$ subanalytic is the same as semianalytic; see the preliminary section), what can be said about the Riemann map from the domain to the unit ball with respect to 'tame' behaviour. Efroymson showed in [15] that any semialgebraic and simply connected domain in the plane is Nash isomorphic to $\mathbb{R}^{2}$. The Riemann map however, is in general not subanalytic as we will see below. It often occurs that the category of (globally) subanalytic sets and maps is too small for concepts from analysis. For example, solutions to ordinary differential equations with subanalytic row data and volume of subanalytic families are in general not subanalytic. But in these two examples the resulting functions can be realized in so-called o-minimal structures (see Comte et al. [4] and Wilkie [33]). O-minimal structures represent an excellent 'tame' generalization of the category of semialgebraic or globally subanalytic sets and functions and are defined by finiteness properties (see Van den Dries [8] and the preliminaries for more details). Under certain conditions on the singular boundary points of the given bounded, semianalytic domain we obtain an o-minimality result:

\section{Theorem A}

There is an o-minimal structure with the following property:

Let $\Omega \subset \mathbb{C}$ be a bounded simply connected domain which is semianalytic. Suppose that the following condition holds: if $x$ is a singular boundary point of $\Omega$ then the angle of the boundary at $x$ is an irrational multiple of $\pi$. Then the Riemann map from $\Omega$ onto the unit ball (i.e. its graph considered as a subset of $\mathbb{R}^{4}$ ) is definable in this o-minimal structure.

We will make the notion of angle at a boundary point more precise in the text. As applications we obtain from Theorem A the following: working with polygons we get the definability of the Schwarz-Christoffel maps, and working with circular polygons we get the definability of certain ratios of hypergeometric functions in this o-minimal structure. Using general model theory the Riemann Mapping Theorem can be transferred by Theorem A to real closed fields of arbitrary large cardinality in a 'tame' way (compare with Peterzil-Starchenko [26, 27] and also with Huber-Knebusch [17] and Knebusch [22] for the development of complex analysis in o-minimal structures on arbitrary real closed fields).

The ideas of the proof are the following: let $\Omega$ be a bounded semianalytic domain in the plane and let $\varphi: \Omega \rightarrow B(0,1)$ be a Riemann map (i.e. a biholomorphic map). Let $x \in \bar{\Omega}$. If $x \in \Omega$ then $\varphi$ is analytic in a neighbourhood of $x$ and if $x \in \partial \Omega$ is a nonsingular boundary point of $\Omega$ (i.e. the boundary is an analytic manifold at $x$ ) then $\varphi$ has an analytic extension to a neighbourhood of $x$ by the Schwarz reflection principle. So the interesting (and hard) case is when $x \in \partial \Omega$ is singular. Taking the inverse of $\varphi$ and composing it with a Möbius transformation (a linear fractional map) it is an equivalent problem to consider a Riemann map $\Phi: \mathbb{H} \rightarrow \Omega$ where $\mathbb{H}$ denotes the upper half plane.

Given a simply connected domain $D$ which has an analytic corner at $0 \in \partial D$ (i.e. the boundary at 0 is given by two regular analytic arcs which intersect in an angle $\varangle D$ greater than 0 ), Lehman showed in [23] (see also Pommerenke [28, p.58]) that a Riemann map $\Phi: \mathbb{H} \rightarrow D$ with $\Phi(0)=0$ 
has an asymptotic development at 0 of the following kind:

$$
\Phi(z) \sim \sum_{n=0}^{\infty} a_{n} P_{n}(\log z) z^{\alpha_{n}} \quad \text { as } \quad z \longrightarrow 0 \quad \text { on } \quad \mathbb{H}
$$

i.e. for each $N \in \mathbb{N}_{0}$ we have

$$
\Phi(z)-\sum_{n=0}^{N} a_{n} P_{n}(\log z) z^{\alpha_{n}}=o\left(z^{\alpha_{N}}\right) \quad \text { as } \quad z \longrightarrow 0 \quad \text { on } \quad \mathbb{H},
$$

where $\alpha_{n} \in \mathbb{R}_{>0}$ with $\alpha_{n} \nearrow \infty, P_{n} \in \mathbb{C}[z]$ monic and $a_{n} \in \mathbb{C}$.

Moreover, if $\varangle D / \pi \in \mathbb{R} \backslash \mathbb{Q}$, then $P_{n}=1$ for all $n \in \mathbb{N}_{0}$. Note that $\alpha_{0}=\varangle D / \pi$ and $P_{0}=1$ for any angle. In particular we see that the Riemann map is not subanalytic if $\varangle D / \pi \in \mathbb{R} \backslash \mathbb{Q}$. To use this asymptotic development we want to have a quasianalytic property; we want to realize these Riemann maps in a class of functions with an asymptotic development as in $(\dagger)$ such that the functions in this class are determined by the (in general not convergent) asymptotic expansion. Such quasianalyticity properties are key tools in generating o-minimal structures (see [21], Van den Dries-Speissegger [11, 12] and Rolin et al. [30]; see also Badalayan [1] for quasianalytic classes of this kind).

Exactly the same kind of asymptotic development occurs at a transition map of a real analytic vector field on $\mathbb{R}^{2}$ at a hyperbolic singularity (see Ilyashenko [18]). Poincaré return maps are compositions of finitely many transition maps and are an important tool to understand qualitatively the trajectories and orbits of a polynomial or analytic vector field on the plane. Following Dulac's approach (see [13]), Ilyashenko uses asymptotic properties of the Poincaré maps to solve Dulac's problem (the weak form of (the second part) of Hilbert's 16th problem): a polynomial vector field on the plane has finitely many limit cycles (see Ilyashenko [19] for an overview of the history of Hilbert 16, part 2). One of the first steps in Ilyashenko's proof is to show that the transition maps at hyperbolic singularities are in a certain quasianalytic class. Formulating his result on the Riemann surface of the logarithm (compare with the introduction of [21] and with [21, Proposition 2.8]) he proves that the considered transition maps have a holomorphic extension to certain subsets of the Riemann surface of the logarithm, so-called standard quadratic domains (see Section 2 below), such that the asymptotic development holds there.

By doing reflections at analytic arcs infinitely often we are able to extend the Riemann map from the upper half plane to a simply connected domain with an analytic corner (at 0 ) to a standard quadratic domain such that the asymptotic development holds there. As a consequence we can show the following

\section{Theorem B}

Let $\Omega \subset \mathbb{C}$ be a bounded simply connected domain which is semianalytic. Let $\Phi: \mathbb{H} \rightarrow \Omega$ be a Riemann map such that 0 is mapped to a boundary point of $\Omega$ with angle different from 0 . Then $\Phi$ can be realized in the quasianalytic class of Ilyashenko described above.

Transition maps at a hyperbolic singularity exhibit a similar dichotomy of the asymptotic development as indicated in $(\dagger)$, depending whether the hyperbolic singularity is resonent or non-resonant, i.e. whether the ratio of the two eigenvalues of the linear part of the vector field at 
the given hyperbolic singularity is rational or irrational, see [13] and [21]. In [21] it is shown that transition maps at non-resonant hyperbolic singularities are definable in a common o-minimal structure, denoted by $\mathbb{R}_{\mathcal{Q}}$. This is obtained by proving that the functions (restricted to the positive line) in Ilyashenko's quasianalytic class which have no log-terms in their asymptotic expansion, generate an o-minimal structure $\mathbb{R}_{\mathcal{Q}}$. Using this result we can derive Theorem A from Theorem B (with the o-minimal structure $\mathbb{R}_{\mathcal{Q}}$ ). Theorem A (and B) are also generalized to the case of unbounded simply connected and globally semianalytic proper domains.

One may think of possible generalizations of Theorem A. In the case of angle different from 0 which is a rational multiple of $\pi$ the Riemann map is realized in the quasianalytic class of Ilyashenko by Theorem B. But so far there is no proof of o-minimality if the asymptotic expansion has logarithmic terms. If the angle is 0 there is no asymptotic development known, although some asymptotic behaviour is known. The proof of Theorem B could be generalized to domains definable in other o-minimal structures (for example the o-minimal structure $\mathbb{R}_{\text {an }}^{*}$, see [11]), but we lose then the dichotomy of the asymptotic development depending whether the angle divided by $\pi$ is irrational or rational, which is essential for the formulation of Theorem A and for the application of the results in [21].

This paper is organized as follows: first, we present in a preliminary section the basic facts about (globally) semi- and subanalytic sets, o-minimal structures, and the Riemann Mapping Theorem which we use throughout the text. In Section 1 we rigorously define what we mean by angle of the boundary at a boundary point of a semianalytic domain and we introduce the concept of domains with an analytic corner. In Section 2 we introduce the quasianalytic class established by Ilyashenko and prove Theorem B in several steps, the main one given by domains with an analytic corner. In Section 3 we obtain Theorem A and give applications.

\section{Notation.}

By $\mathbb{N}$ we denote the set of natural numbers and by $\mathbb{N}_{0}$ the set of nonnegative integers. Let $a \in \mathbb{C}$ and $r>0$. We set $B(a, r):=\{z \in \mathbb{C}|| z-a \mid<r\}$ and $\bar{B}(a, r):=\{z \in \mathbb{C}|| z-a \mid \leq r\}$, where || is the euclidean norm. A domain is an open, nonempty and connected set (in a topological space). A domain in $\mathbb{C}$ is called simply connected if its complement has no bounded connected components. So a bounded domain in $\mathbb{C}$ is simply connected iff its complement is connected. Given an open set $U$ of a Riemann surface we denote with $\mathcal{O}(U)$ the $\mathbb{C}$-algebra of holomorphic functions on $U$ with values in $\mathbb{C}$. We identify $\mathbb{C}$ with $\mathbb{R}^{2}$. 
THE RIEMANN MAPPING THEOREM FOR SEMIANALYTIC DOMAINS AND O-MINIMALITY

\section{Preliminaries}

\section{a) Semi- and subanalytic sets}

A subset $A$ of $\mathbb{R}^{n}, n \geq 1$, is called semianalytic if the following holds:

for each $x_{0} \in \mathbb{R}^{n}$ there are open neighbourhoods $U, V$ of $x_{0}$ with $\bar{U} \subset V$ and there are realanalytic functions $f_{i}, g_{i, 1}, \ldots, g_{i, k}$ on $V, 1 \leq i \leq \ell$, such that

$$
A \cap U=\bigcup_{1 \leq i \leq \ell}\left\{x \in U \mid f_{i}(x)=0, g_{i, 1}(x)>0, \ldots, g_{i, k}(x)>0\right\} .
$$

A subset $B$ of $\mathbb{R}^{n}, n \geq 1$, is called subanalytic if the following holds:

for each $x_{0} \in \mathbb{R}^{n}$ there is an open neighbourhood $U$ of $x_{0}$, some $m \geq n$ and some bounded semianalytic set $A \subset \mathbb{R}^{m}$ such that $B \cap U=\pi_{n}(A)$ where $\pi_{n}: \mathbb{R}^{m} \rightarrow \mathbb{R}^{n},\left(x_{1}, \ldots, x_{m}\right) \mapsto$ $\left(x_{1}, \ldots, x_{n}\right)$, is the projection on the first $n$ coordinates.

A map is called semianalytic resp. subanalytic if its graph is a semianalytic resp. subanalytic set. A set is called globally semianalytic resp. globally subanalytic if it is semianalytic resp. subanalytic after applying the semialgebraic homeomorphism $\left.\mathbb{R}^{n} \rightarrow\right]-1,1\left[{ }^{n}, x_{i} \mapsto x_{i} / \sqrt{1+x_{i}^{2}}\right.$, (or equivalently if it is semianalytic resp. subanalytic in the ambient projective space, see [7] and $[10$, pp.505-506]).

Semi- and subanalytic sets exhibit nice 'tame' behaviour (see for example [2], [6], [24], and [31]). One-dimensional (globally) subanalytic sets and (globally) subanalytic subsets of $\mathbb{R}^{2}$ are (globally) semianalytic (see [2, Theorem 6.1]). A bounded semianalytic function on the positive

real line is given locally at 0 by a convergent Puiseux series $\sum_{n=0}^{\infty} a_{n} t^{\frac{n}{d}}$ for some $d \in \mathbb{N}$ (see for example [7, p.192]).

\section{b) O-minimal structures}

O-minimal structures are axiomatically defined as follows.

For $n \in \mathbb{N}$ let $M_{n}$ be a set of subsets of $\mathbb{R}^{n}$ and let $\mathcal{M}:=\left(\mathcal{M}_{n}\right)_{n \in \mathbb{N}}$. Then $\mathcal{M}$ is called a structure on $\mathbb{R}$, if the following axioms hold for each $n \in \mathbb{N}$ :

(S1) $\mathcal{M}_{n}$ is a boolean algebra of subsets of $\mathbb{R}^{n}$ with $\mathbb{R}^{n} \in \mathcal{M}_{n}$.

(S2) If $A \in \mathcal{M}_{n}$, then $\mathbb{R} \times A$ and $A \times \mathbb{R}$ belong to $\mathcal{M}_{n+1}$.

(S3) $\left\{\left(x_{1}, \ldots, x_{n}\right) \in \mathbb{R}^{n} \mid x_{1}=x_{n}\right\} \in \mathcal{M}_{n}$ for $n>0$.

(S4) If $A \in \mathcal{M}_{n+1}$, then $\pi(A) \in \mathcal{M}_{n}$, where $\pi: \mathbb{R}^{n+1} \rightarrow \mathbb{R}^{n}$ is the projection on the first $n$ coordinates.

An o-minimal structure on $\mathbb{R}$ is a structure $\mathcal{M}$ on $\mathbb{R}$ with the additional properties

(O0) $\{r\} \in \mathcal{M}_{1}$ for all $r \in \mathbb{R}$.

(O1) $\left\{(x, y) \in \mathbb{R}^{2} \mid x<y\right\} \in \mathcal{M}_{2}$.

(O2) The sets in $\mathcal{M}$ are exactly the finite unions of intervals and points.

Here $<$ is the canonical order on $\mathbb{R}$. An o-minimal structure $\mathcal{M}$ on $\mathbb{R}$ expands the field $\mathbb{R}$ if the following holds:

(R1) $\left\{(x, y, z) \in \mathbb{R}^{3} \mid z=x+y\right\} \in \mathcal{M}_{3}$.

(R2) $\left\{(x, y, z) \in \mathbb{R}^{3} \mid z=x \cdot y\right\} \in \mathcal{M}_{3}$. 
A subset of $\mathbb{R}^{n}$ is called definable in the structure $\mathcal{M}$ on $\mathbb{R}$ if it belongs to $M_{n}$. A function is definable in $\mathcal{M}$ if its graph is definable in $\mathcal{M}$. Axiom (O2) implies that a subset of $\mathbb{R}$, definable in an o-minimal structure on $\mathbb{R}$, has finitely many components. Axiom (R1) and (R2) imply that addition and multiplication are definable in an o-minimal structure expanding the field $\mathbb{R}$.

Not only definable subsets of $\mathbb{R}$ have finitely many connected components, much more can be deduced from the axioms of o-minimality: a definable subset of $\mathbb{R}^{n}, n \in \mathbb{N}$ arbitrary, has finitely many connected components that are again definable. If the o-minimal structure expands the field $\mathbb{R}$ then definable sets can be definably triangulated and have a definable $C^{k}$-stratification for any $k \in \mathbb{N}$. Hence for a given $k \in \mathbb{N}$, a definable function is $C^{k}$ outside a definable set of small dimension. General facts about o-minimal structures can be found in [8].

\section{Examples of o-minimal structures on the field $\mathbb{R}$ :}

(i) There is a 'smallest' (with respect to inclusion of the boolean algebras of definable sets in any dimension) o-minimal structure on the field $\mathbb{R}$ denoted by $(\mathbb{R},+, \cdot,<)$. The definable sets are exactly the semialgebraic sets, i.e. a definable subset of $\mathbb{R}^{n}$ is a finite union of sets of the form

$$
\left\{x \in \mathbb{R}^{n} \mid f(x)=0, g_{1}(x)>0, \ldots, g_{r}(x)>0\right\}
$$

with $f, g_{1}, \ldots, g_{r} \in \mathbb{R}\left[X_{1}, \ldots, X_{n}\right]$ (see [3] for more details).

Given a structure $\mathcal{M}$ and functions $f_{j}: \mathbb{R}^{n_{j}} \rightarrow \mathbb{R}(j$ is in some index set $J)$, we denote by $\mathcal{M}\left(\left(f_{j}\right)_{j \in J}\right)$ the 'smallest' structure which contains all sets definable in $\mathcal{M}$ and the graphs of all functions $f_{j}$.

(ii) $\mathbb{R}_{\exp }=\left(\mathbb{R},+, \cdot,<\right.$, exp) where exp is the exponential function exp: $\mathbb{R} \rightarrow \mathbb{R}_{>0}$ (see [33] for more details).

(iii) $\mathbb{R}_{\mathrm{an}}=(\mathbb{R},+, \cdot,<,(f))$ where $f$ ranges over all the restricted analytic functions. A function $f: \mathbb{R}^{n} \rightarrow \mathbb{R}$ is called restricted analytic if the following holds:

$$
f=\left\{\begin{array}{lll}
g & \text { on }[-1,1]^{n}, & g \text { analytic on a neighbourhood } \\
0 & \text { outside }[-1,1]^{n} . & \text { of }[-1,1]^{n},
\end{array}\right.
$$

The sets definable in $\mathbb{R}_{\mathrm{an}}$ are exactly the globally subanalytic sets and the bounded sets definable in $\mathbb{R}_{\mathrm{an}}$ are exactly the bounded subanalytic sets (see [7] and [10, p.505] for more details).

(iv) $\mathbb{R}_{\mathrm{an}}^{\mathbb{R}}=\mathbb{R}_{\mathrm{an}}\left(\left(x^{r}\right)_{r \in \mathbb{R}}\right)$ where $x^{r}$ is given by

$$
x^{r}: \mathbb{R} \longrightarrow \mathbb{R}, x \longmapsto\left\{\begin{array}{cc}
a^{r} & , \quad a>0, \\
0 & , \quad a \leq 0,
\end{array}\right.
$$

(see Miller [25] for more details).

(v) $\mathbb{R}_{\text {an }, \exp }=\mathbb{R}_{\text {an }}(\exp )$ where exp is the exponential function exp: $\mathbb{R} \rightarrow \mathbb{R}_{>0}$ (see Van den Dries et al. [9] for more details).

(vi) $\mathbb{R}_{\text {an }}^{*}$, the o-minimal structure in which convergent generalized real power series are definable (see [11] for more details). 
(vii) $\mathbb{R}_{\mathcal{Q}}$, the o-minimal structure in which transition maps of real analytic vector fields on the plane at non-resonant hyperbolic singularities are definable (see [21] for more details).

\section{c) Riemann Mapping Theorem}

Let $\Omega \varsubsetneqq \mathbb{C}$ be a simply connected domain. Then $\Omega$ can be mapped biholomorphically onto the unit ball $B(0,1)=\{z \in \mathbb{C}|| z \mid<1\}$.

Such a biholomorphic map (from a simply connected domain onto the unit ball) is not totally unique, but the following holds (see [29, p.179]):

\section{Uniqueness}

Let $a \in \Omega$. Then there is exactly one biholomorphic map $\varphi: \Omega \rightarrow B(0,1)$ with $\varphi(a)=0$ and $\varphi^{\prime}(a)>0$.

\section{Examples}

(i) The group of holomorphic automorphisms of the unit ball is given by

$$
\text { Aut } B(0,1)=\left\{z \longmapsto \rho \frac{z-a}{\bar{a} z-1}|| a|<1,| \rho \mid=1\right\} .
$$

(ii) Let $\mathbb{H}:=\{z \in \mathbb{C} \mid \operatorname{Im} z>0\}$ be the upper half plane. Then $\mathbb{H} \stackrel{\cong}{\longrightarrow} B(0,1), z \longmapsto \frac{z-i}{z+i}$, is a biholomorphic map onto the unit ball.

The maps from the example above are all fractional linear maps, so-called Möbius transformations (see Conway [5, III. §3]). With the uniqueness result above we see that the Möbius transformations are exactly the biholomorphic maps between sets which are either balls or open half planes. Möbius transformations are semialgebraic (identifying $\mathbb{C}$ with $\mathbb{R}^{2}$ ) and are therefore definable in every o-minimal structure (expanding the field $\mathbb{R}$ ). A biholomorphic map from one simply connected domain onto a second one will be here referred to as a Riemann map. Having one Riemann map from a simply connected domain onto the unit ball you get all of them by composing with the Möbius transformations, which are automorphisms of the unit ball.

Let $\Omega \subset \mathbb{C}$ be a bounded and semianalytic domain. Then since the o-minimal structure $\mathbb{R}_{\mathrm{an}}$ has analytic cell decomposition (see [10, pp.508-509]), the boundary is real analytic at all but finitely many boundary points. Given a boundary point $x \in \partial \Omega$ there is $k \in \mathbb{N}$ such that for all $r>0$ small enough, $\Omega \cap B(x, r)$ consists of $k$ connected components (each semianalytic) such that each of them is a Jordan domain. A Jordan domain is a domain whose boundary is a closed Jordan curve. A Jordan domain is simply connected. Let $C$ be one of the connected components of $\Omega \cap B(x, r), r>0$ small. If $\Omega$ is simply connected and $\varphi$ a Riemann map onto a simply connected bounded and semianalytic domain $\Omega^{\prime}$, then by the Curve Selection Lemma (see [8, p.94]) and Carathéodory's Prime End Theorem (see [28, Chapter 2 p.18]), $\varphi$ has a continuous extension to $\bar{C}$ with $\varphi(\bar{C} \cap \partial \Omega) \subset \partial \Omega^{\prime}$. 


\section{Angles and domains with an analytic corner}

Let $\Omega$ be a bounded and subanalytic domain in $\mathbb{R}^{n}$. Let $x \in \partial \Omega:=\bar{\Omega} \backslash \Omega$. Then the germ of $\Omega$ at $x$ has finitely many connected components. More precisely we have the following: there is $k \in \mathbb{N}$ such that for all sufficiently small neighbourhoods $V$ of $x$ the set $\Omega \cap V$ has exactly $k$ components having $x$ as boundary point.

Let $\Omega \subset \mathbb{R}^{2}$ be a bounded and semianalytic domain without isolated boundary points. Let $x \in \partial \Omega$ and let $C$ be a connected component of the germ of $\Omega$ at $x$. Then the germ of the boundary of $C$ at $x$ is given by (the germs of) two semianalytic curves. So the interior angle

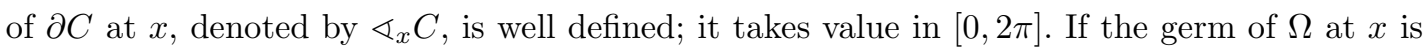
connected we write $\varangle_{x} \Omega$.

\section{Definition 1.1.}

Let $\Omega \subset \mathbb{R}^{2}$ be a bounded semianalytic domain without isolated boundary points.

a) A point $x \in \partial \Omega$ is a singular boundary point if $\partial \Omega$ is not a real analytic manifold at $x$.

b) We set $\operatorname{Sing}(\partial \Omega):=\{x \in \partial \Omega \mid x$ is a singular boundary point of $\partial \Omega\}$.

c) Let $x \in \partial \Omega$. We set $\varangle(\Omega, x):=\left\{\varangle_{x} C \mid C\right.$ is a component of the germ of $\Omega$ at $x$ and $x \in \operatorname{Sing}(\partial C)\}$.

\section{Remark 1.2.}

Let $\Omega \subset \mathbb{R}^{2}$ be a bounded semianalytic domain without isolated boundary points.

a) Then $\operatorname{Sing}(\partial \Omega)$ is finite by analytic cell decomposition (see [10, pp.508-509]).

b) Let $x \in \partial \Omega$. Then $\varangle(\Omega, x)=\emptyset$ iff $x \notin \operatorname{sing}(\partial C)$ for all components $C$ of the germ of $\Omega$ at $x$. This is especially the case if $x \notin \operatorname{Sing}(\partial \Omega)$.

\section{Example 1.3.}

a) We consider $\Omega:=B(0,1) \backslash\left[-\frac{1}{2}, \frac{1}{2}\right]$. Then $\partial \Omega=\partial B(0,1) \cup\left[-\frac{1}{2}, \frac{1}{2}\right]$ and $\operatorname{Sing}(\partial \Omega)=\left\{-\frac{1}{2}, \frac{1}{2}\right\}$. Given $x \in \partial B(0,1)$ the germ of $\Omega$ at $x$ has one component and we have $\varangle_{x} \Omega=\pi$. Given $x \in]-\frac{1}{2}, \frac{1}{2}\left[\right.$ the germ of $\Omega$ at $x$ has two components $C_{1}, C_{2}$, and we have $\varangle{ }_{x} C_{1}=\varangle_{x} C_{2}=$ $\pi$. For $x= \pm \frac{1}{2}$ the germ of $\Omega$ at $x$ has one component $C_{ \pm}$with $\varangle_{ \pm \frac{1}{2}} C_{ \pm}=2 \pi$. Hence $\varangle\left(\Omega, \pm \frac{1}{2}\right)=\{2 \pi\}$.

b) We consider the simply connected domain $\Omega:=B(1,1) \backslash \bar{B}\left(\frac{1}{2}, \frac{1}{2}\right)$. Then $0 \in \operatorname{Sing}(\partial \Omega)$ and the germ of $\Omega$ at 0 has two components. We have $\varangle(\Omega, 0)=\{0\}$.

\section{Definition 1.4.}

Let $\Omega \varsubsetneqq \mathbb{R}^{2}$ be a (not necessarily bounded) globally semianalytic domain (or equivalently, definable in the o-minimal structure $\mathbb{R}_{\text {an }}$ ) without isolated boundary points.

a) By $\partial^{\infty} \Omega$ we denote the boundary of $\Omega$ with respect to the standard topology in $\mathbb{R}^{2} \cup\{\infty\}$.

b) We set $\Omega^{\prime}:=\frac{1}{\Omega} \cap B(0,1)$, where $\frac{1}{\Omega}:=\left\{\frac{1}{z} \mid z \in \Omega \backslash\{0\}\right\}$.

(i) We define $\operatorname{Sing}(\partial \Omega)$ as follows: Let $x \in \partial^{\infty} \Omega$. If $x \neq \infty$ then $x \in \operatorname{Sing}(\partial \Omega)$ iff $x \in$ $\operatorname{Sing}(\partial(\Omega \cap B(0,|x|+1)))$. If $x=\infty$ we have $\infty \in \operatorname{Sing}(\partial \Omega)$ iff $0 \in \operatorname{Sing}\left(\partial \Omega^{\prime}\right)$.

(ii) Let $x \in \partial^{\infty} \Omega$ and let $C$ be a component of the germ of $\Omega$ at $x$. If $x \neq \infty$ then $C$ is also a germ of $\Omega \cap B(0,|x|+1)$ at $x$ and we define $\varangle_{x} C$ as in Remark 1.2. If $x=\infty$ we set $\varangle_{\infty} C:=\varangle_{0} C^{\prime}$ with $C^{\prime}:=\frac{1}{C} \cap B(0,1)$. 
(iii) Let $x \in \partial^{\infty} \Omega$. If $x \neq \infty$ we set $\varangle(\Omega, x):=\varangle(\Omega \cap B(0,|x|+1))$. If $x=\infty$ we set $\varangle(\Omega, \infty):=\varangle\left(\Omega^{\prime}, 0\right)$.

Let $\Omega$ be a semianalytic and simply connected bounded domain. Let $\Phi: \mathbb{H} \rightarrow \Omega$ be a Riemann map. By Carathéodory's Prime End Theorem we know that $\Phi$ has a continuous extension to $\overline{\mathbb{H}}$ (see part c) of the preliminary section). Let $x:=\Phi(0) \in \partial \Omega$. Then there is a component $C$ of the germ of $\Omega$ at $x$ such that the germ of $\mathbb{H}$ at 0 is mapped conformally to $C$ by $\Phi$. We say that 0 is mapped by $\Phi$ to $x$ with attached angle $\varangle_{x} C$.

Definition 1.5 (compare with [23]).

We say that a domain $D \subset \mathbb{C}$ with $0 \in \partial D$ has an analytic corner (at 0 ) if the boundary of $D$ at 0 is given by two analytic arcs which are regular at 0 and if $D$ has an interior angle greater than 0 . More precisely, the following holds:

There are holomorphic functions $\varphi_{(1)}, \varphi_{(2)} \in \mathcal{O}(B(0,1))$ with $\varphi_{(1)}(0)=\varphi_{(2)}(0)=0$ and $\varphi_{(1)}^{\prime}(0)$. $\varphi_{(2)}^{\prime}(0) \neq 0$ such that with $\Gamma_{1}:=\varphi_{(1)}\left(\left[0,1[)\right.\right.$ and $\Gamma_{2}:=\varphi_{(2)}([0,1[)$ the following holds:

a) $\partial D \cap B(0, r)=\left(\Gamma_{1} \cup \Gamma_{2}\right) \cap B(0, r)$ for some $r>0$.

b) The interior angle $\varangle D \in[0,2 \pi]$ of $\partial D$ at 0 is greater than 0 .

Note that possibly $\Gamma_{1}=\Gamma_{2}$ if $\varangle D=2 \pi$. Otherwise we may assume that $\Gamma_{1} \cap \Gamma_{2}=\{0\}$.

\section{Remark 1.6.}

Let $D \subset \mathbb{C}$ be a simply connected domain with an analytic corner at $0 \in \partial D$. Let $\Phi: \mathbb{H} \rightarrow D$ be a Riemann map. Again by Carathéodory's Prime End Theorem (see part c) of the preliminary section) we get the following: the inverse $\Phi^{-1}$ has a continuous extension to the boundary $\partial D$ in a neighbourhood of $0 \in \partial D$ with $\Phi^{-1}(0)=: x \in \partial \mathbb{H}$ and $\Phi$ has a continuous extension to the boundary $\partial \mathbb{H}$ in a neighbourhood of $x \in \partial \mathbb{H}$ with $\Phi(x)=0$. Choosing an automorphism $\tau$ of $\mathbb{H}$ (a certain Möbius transformation) which maps 0 to $x$ and replacing $\Phi$ by $\Phi \circ \tau$ we can assume that $\Phi(0)=0$. Renaming the indices $i=1,2$ from Definition 1.5 we can assume that the germ of $\mathbb{R}_{\geq 0}$ at 0 is mapped by $\Phi$ to the germ of $\Gamma_{1}$ at 0 and that the germ of $\mathbb{R}_{\leq 0}$ at 0 is mapped by $\Phi$ to the germ of $\Gamma_{2}$ at 0 . We say that the positive direction is mapped to $\Gamma_{1}$ and the negative direction is mapped to $\Gamma_{2}$.

\section{Riemann maps at semianalytic domains and a quasianalytic class of Ilyashenko}

Lehman showed in [23] that the Riemann map has an asymptotic development at an analytic corner (see also [28, p.58]; Wigley [32] showed the existence of asymptotic development for more general corners). We introduce the series which occur as asymptotic expansions.

Definition 2.1 (compare with [21, Section 1]).

Let $z$ be an indeterminate. A generalized log-power series in $z$ is a formal expression $g(z)=$ $\sum_{\alpha \in \mathbb{R}_{\geq 0}} a_{\alpha} P_{\alpha}(\log z) z^{\alpha}$ with $a_{\alpha} \in \mathbb{C}$ and $P_{\alpha} \in \mathbb{C}[z] \backslash\{0\}$ monic with $P_{0}=1$ such that the support 
of $g$, defined as $\operatorname{supp}(g):=\left\{\alpha \in \mathbb{R}_{\geq 0} \mid a_{\alpha} \neq 0\right\}$, fulfils the following condition: for all $R>0$ the set $\operatorname{supp}(g) \cap[0, R]$ is finite. We write $\mathbb{C}\left[\left[z^{*}\right]\right]_{\log }^{\omega}$ for the set of generalized log-power series. For $g \in \mathbb{C}\left[\left[z^{*}\right]\right]_{\log }^{\omega}$ we set $\nu(g):=\min \operatorname{supp}(g)$. By $\mathbb{C}\left[\left[z^{*}\right]\right]^{\omega}$ we denote the subset of $\mathbb{C}\left[\left[z^{*}\right]\right]_{\log }^{\omega}$ consisting of all $g \in \mathbb{C}\left[\left[z^{*}\right]\right]_{\log }^{\omega}$ with $P_{\alpha}=1$ for all $\alpha \in \mathbb{R}_{\geq 0}$. By $\mathbb{C}\left[\left[z^{*}\right]\right]_{\log }^{\omega, \text { fin }}$ (resp. $\mathbb{C}\left[\left[z^{*}\right]\right]^{\omega, \text { fin }}$ ) we denote the set of all $g \in \mathbb{C}\left[\left[z^{*}\right]\right]_{\log }^{\omega}\left(\right.$ resp. $\left.\mathbb{C}\left[\left[z^{*}\right]\right]^{\omega}\right)$ with finite support.

Convention. From now on we omit the superscript $\omega$.

\section{Remark 2.2.}

a) The set $\mathbb{C}\left[\left[z^{*}\right]\right]_{\log }$ is in a natural way a $\mathbb{C}$-algebra with $\mathbb{C}\left[\left[z^{*}\right]\right]$ as subalgebra.

b) Interpreting $\log$ as the principal branch of the logarithm, i.e. as $\log : \mathbb{C} \backslash \mathbb{R}_{\leq 0} \rightarrow \mathbb{C}, z \mapsto$ $\log |z|+i \operatorname{Arg} z$, (with $\operatorname{Arg}(z) \in]-\pi, \pi\left[\right.$ the standard argument) and $z^{\alpha}$ as the power function $z^{\alpha}: \mathbb{C} \backslash \mathbb{R}_{\leq 0} \rightarrow \mathbb{C}, z \mapsto \exp (\alpha \log z)$, we get that $g \in \mathcal{O}\left(\mathbb{C} \backslash \mathbb{R}_{\leq 0}\right)$ if $g \in \mathbb{C}\left[\left[z^{*}\right]\right]_{\log }^{\text {fin }}$.

\section{Definition 2.3.}

Let $f \in \mathcal{O}(\mathbb{H})$ and let $g=\Sigma a_{\alpha} P_{\alpha}(\log z) z^{\alpha} \in \mathbb{C}\left[\left[z^{*}\right]\right]_{\log }$. We say that $f$ has asymptotic expansion $g$ on $\mathbb{H}$ and write $f \sim_{\mathbb{H}} g$, if for each $R>0$

$$
f(z)-\sum_{\alpha \leq R} a_{\alpha} P_{\alpha}(\log z) z^{\alpha}=o\left(|z|^{R}\right) \quad \text { as } \quad|z| \longrightarrow 0 \quad \text { on } \quad \mathbb{H} .
$$

Note that $g$ is unique.

Fact 2.4 (see Lehman [23, Theorem 1]).

Let $D$ be a simply connected domain with an analytic corner and let $\Phi: \mathbb{H} \rightarrow D$ be a Riemann map with $\Phi(0)=0$. Then there is $g \in \mathbb{C}\left[\left[z^{*}\right]\right]_{\log }$ such that $f \sim_{\mathbb{H}} g$. If $\varangle D / \pi \in \mathbb{R} \backslash \mathbb{Q}$ then $g \in \mathbb{C}\left[\left[z^{*}\right]\right]$.

In the above situation we have with $\alpha:=\varangle D / \pi$ that $\operatorname{supp}(g) \subset \mathbb{N}_{0}+\mathbb{N} \alpha$, that $\nu(g)=\alpha$ and that $P_{\nu(g)}=1$. Moreover, by Remark 1.6 the Riemann map has a continuous extension to the boundary of $\mathbb{H}$ near 0 . The estimates of Definition 2.3 then hold on $\overline{\mathbb{H}} \cap B(0, r)$ for some $r>0$ (with the continuous extension of the logarithm and the power functions to $\overline{\mathbb{H}}$ respectively), see [23, Section 4].

We want to realize the Riemann map in the quasianalytic class used by Ilyashenko in his work on Hilbert 16. For this we have to consider holomorphic functions on the Riemann surface of the logarithm (compare with [21, Section 2]):

\section{Definition 2.5.}

We define the Riemann surface of the logarithm $\mathbf{L}$ in polar coordinates by $\mathbf{L}:=\mathbb{R}_{>0} \times \mathbb{R}$. Then $\mathbf{L}$ is a Riemann surface with the isomorphic holomorphic projection map $\log : \mathbf{L} \rightarrow \mathbb{C}$, $(r, \varphi) \mapsto \log r+i \varphi$. For $z=(r, \varphi) \in \mathbf{L}$ we define the absolute value by $|z|:=r$ and the $\operatorname{argument}$ by $\arg z:=\varphi$. For $r>0$ we set $B_{\mathbf{L}}(r):=\{z \in \mathbf{L}|| z \mid<r\}$. We identify $\mathbb{C} \backslash \mathbb{R}_{\leq 0}$ with $\left.\mathbb{R}_{>0} \times\right]-\pi, \pi\left[\subset \mathcal{L}\right.$ via polar coordinates. Let $\alpha \geq 0$. We define the power function $z^{\alpha}$ as $z^{\alpha}: \mathbf{L} \rightarrow \mathbb{C}, z=(r, \varphi) \mapsto \exp (\alpha \log z)$. For each real $\rho>0$, the map $\mathbf{p}: \mathbf{L} \rightarrow \mathbf{L}$ is defined by $\mathbf{p}^{\rho}(r, \varphi):=\left(r^{\rho}, \rho \varphi\right)$, and the map $\mathbf{m}: \mathbf{L}^{2} \rightarrow \mathbf{L}$ is defined by $\mathbf{m}\left(\left(r_{1}, \varphi_{1}\right),\left(r_{2}, \varphi_{2}\right)\right):=\left(r_{1} r_{2}, \varphi_{1}+\right.$ $\left.\varphi_{2}\right)$ (see $[21$, Section 4]). 
THE RIEMANN MAPPING THEOREM FOR SEMIANALYTIC DOMAINS AND O-MINIMALITY 11

\section{Definition 2.6.}

A domain $W \subset \mathbf{L}$ of the Riemann surface of the logarithm is a standard quadratic domain if there are constants $c, C>0$ such that

$$
W=\{(r, \varphi) \in \mathbf{L} \mid 0<r<c \exp (-C \sqrt{|\varphi|})\} .
$$

A domain is called a quadratic domain if it contains a standard quadratic domain.

\section{Definition 2.7.}

Let $U \subset \mathbf{L}$ be a quadratic domain, let $f \in \mathcal{O}(U)$ and let $g=\sum_{\alpha \geq 0} a_{\alpha} P_{\alpha}(\log z) z^{\alpha} \in \mathbb{C}\left[\left[z^{*}\right]\right]_{\log }$. We say that $f$ has asymptotic expansion $g$ on $U$ and write $f \sim_{U} g$, if for each $R>0$ there is a quadratic domain $U_{R} \subset U$ such that

$$
f(z)-\sum_{\alpha \leq R} a_{\alpha} P_{\alpha}(\log z) z^{\alpha}=o\left(|z|^{R}\right) \quad \text { as } \quad|z| \longrightarrow 0 \quad \text { on } \quad U_{R}
$$

We write $T f:=g$. By $\mathcal{Q}^{\log }(U)$ we denote the set of all $f \in \mathcal{O}(U)$ with an asymptotic expansion. By $\mathcal{Q}(U)$ we denote the subset of all $f \in \mathcal{Q}^{\log }(U)$ with $T f \in \mathbb{C}\left[\left[z^{*}\right]\right]$.

\section{Remark 2.8.}

a) Logarithm and power functions on $\mathbf{L}$ extend logarithm and power functions on $\mathbb{C} \backslash \mathbb{R}_{\leq 0}$ (compare with Remark 2.2). Given $g \in \mathbb{C}\left[\left[z^{*}\right]\right]_{\log }^{\text {fin }}$ we get that $g \in \mathcal{O}(\mathbf{L})$. Especially in the situation of Definition 2.7 we get for $R>0$ that $\sum_{\alpha \leq R} a_{\alpha} P_{\alpha}(\log z) z^{\alpha} \in \mathcal{O}(\mathbf{L})$.

b) If $f \in \mathcal{Q}^{\log }(U)$ for some quadratic domain $U$ then there is exactly one $g \in \mathbb{C}\left[\left[z^{*}\right]\right] \log$ with $f \sim_{U} g$; i.e. $T f$ is well defined.

\section{Definition 2.9.}

We define an equivalence relation $\equiv$ on $\underset{U \subset \mathbf{L} \text { quadr. }}{\bigcup} \mathcal{Q}^{\log }(U)$ as follows: $f_{1} \equiv f_{2}$ if and only if there is a quadratic domain $V \subset \mathbf{L}$ such that $\left.f_{1}\right|_{V}=\left.f_{2}\right|_{V}$. We let $\mathcal{Q}^{\log }$ be the set of all $\equiv$-equivalence classes. In the same way we obtain the class $\mathcal{Q}$. Note that $\mathcal{Q}=\mathcal{Q}_{1}^{1}$ in the notation of $[21$, Definition 5.1, Remarks 5.2 \& Definition 5.4].

\section{Remark 2.10.}

a) We will not distinguish between $f \in \bigcup_{U \subset \mathbf{L} \text { quadr. }} \mathcal{Q}^{\log }(U)$ and its equivalence class in $\mathcal{Q}^{\log }$, which we also denote by $f$. Thus $\mathcal{Q}^{\log }(U) \subset \mathcal{Q}^{\log }$ given a quadratic domain $U \subset \mathbf{L}$.

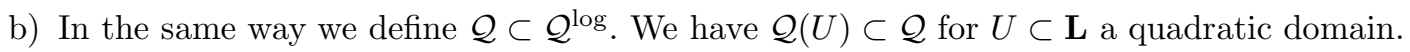

c) Given a quadratic domain $U \subset \mathbf{L}$ the set $\mathcal{Q}^{\log }(U)$ is a $\mathbb{C}$-algebra with $\mathcal{Q}(U)$ as a subalgebra. Also, $\mathcal{Q}^{\log }$ is an algebra with $\mathcal{Q}$ as a subalgebra.

d) Given a quadratic domain $U \subset \mathbf{L}$ the well defined maps $T: \mathcal{Q}^{\log }(U) \rightarrow \mathbb{C}\left[\left[z^{*}\right]\right]_{\log }, f \mapsto T f$, and $T: \mathcal{Q}(U) \rightarrow \mathbb{C}\left[\left[z^{*}\right]\right], f \mapsto T f$, are homomorphisms of $\mathbb{C}$-algebras. Also the induced maps $T: \mathcal{Q}^{\log } \rightarrow \mathbb{C}\left[\left[z^{*}\right]\right]_{\log }, f \mapsto T f$, and $T: \mathcal{Q} \rightarrow \mathbb{C}\left[\left[z^{*}\right]\right], f \mapsto T f$, are homomorphisms of $\mathbb{C}$-algebras. 


\section{Proposition 2.11.}

Let $U \subset \mathbf{L}$ be a quadratic domain. The homomorphism $T: \mathcal{Q}^{\log }(U) \rightarrow \mathbb{C}\left[\left[z^{*}\right]\right]_{\log }$ is injective. Therefore, the homomorphism $T: \mathcal{Q}^{\log } \rightarrow \mathbb{C}\left[\left[z^{*}\right]\right]_{\log }$ is injective.

Proof:

See Ilyashenko [18, Theorem 2 p.23] and [21, Proposition 2.8].

Proposition 2.11 contains the necessary quasianalyticity we need for our o-minimality result. We realize now the Riemann map from the upper half plane to a bounded semianalytic domain with attached angle greater than 0 in this quasianalytic class. Theorem B gets the following precise form:

\section{Theorem 2.12.}

Let $\Omega \subset \mathbb{C}$ be a semianalytic and simply connected bounded domain. Let $\Phi: \mathbb{H} \rightarrow \Omega$ be a Riemann map such that 0 is mapped to a boundary point with attached angle $\varangle$ greater than 0 . Then there is a quadratic domain $U \subset \mathbf{L}$ such that $\Phi \in \mathcal{Q}^{\log }(U)$. If $\varangle \in \pi(\mathbb{R} \backslash \mathbb{Q})$ then $\Phi \in \mathcal{Q}(U)$.

We will prove this theorem in several steps, considering first domains with an analytic corner. We obtain the extension of the Riemann map to a quadratic domain of the Riemann surface of the logarithm by performing reflections at analytic arcs infinitely often. These reflections are obtained by iteration, inversion and conjugation of certain holomorphic functions. To get the desired properties of Definition 2.7 we have to control very carefully this discrete dynamical system. Therefore we use the theory of univalent functions, especially Koebe's $\frac{1}{4}$-Theorem and the Growth Theorem (see for example Duren [14, Chapter 2]). To motivate the technical statements of the upcoming proofs we give the following example for the Schwarz reflection principle at analytic arcs (which reduces to the Schwarz reflection principle at the real line, see [5, IX. 1.1]):

\section{Example 2.13.}

Let $r>0$ and let $V:=\mathbb{H} \cap B(0, r)$. Let $f \in \mathcal{O}(V)$ have the following property: $f$ has a continuous extension to $[0, r[$ and there is an injective holomorphic function $\varphi: B(0,1) \rightarrow \mathbb{C}$ such that $f\left(\left[0, r[) \subset \Gamma:=\varphi\left(\left[0,1[)\right.\right.\right.\right.$. Then there is some $0<r^{\prime} \leq r$ such that $f$ has a holomorphic extension to $B\left(0, r^{\prime}\right) \backslash \mathbb{R}_{\leq 0}$, given by

$$
f: B\left(0, r^{\prime}\right) \backslash \mathbb{R}_{\leq 0} \longrightarrow \mathbb{C}, z \longmapsto\left\{\begin{array}{ccc}
f(z) & \operatorname{Im} z \geq 0, \\
\frac{}{\varphi\left(\varphi^{-1} f(\bar{z})\right)} & \text { if } & \operatorname{Im} z<0 .
\end{array}\right.
$$

\section{Definition 2.14.}

Let $k \in \mathbb{N}_{0}$. We define $T_{k}:=\left\{(r, \varphi) \in \mathbf{L} \mid 0 \leq \varphi \leq 2^{k} \pi\right\}$. For $k \in \mathbb{N}$ we set $T_{k}^{\prime}:=\{(r, \varphi) \in$ $\left.\mathbf{L} \mid 2^{k-1} \pi \leq \varphi \leq 2^{k} \pi\right\}$. Given $k \in \mathbb{N}_{0}$ we define the reflections $\tau_{k}: T_{k+1}^{\prime} \rightarrow T_{k},(r, \varphi) \mapsto(r,-\varphi+$ $\left.2^{k+1} \pi\right)$. Note that $T_{0}=\overline{\mathbb{H}} \backslash\{0\}, T_{k+1}=T_{k} \cup T_{k+1}^{\prime}$ and $\tau_{k}\left(r, 2^{k} \pi\right)=\left(r, 2^{k} \pi\right), \tau_{k}\left(r, 2^{k+1} \pi\right)=$ $(r, 0)$. 
Lemma 2.15.

a) Let $k \geq 0$. Then $\overline{\log \circ \tau_{k}}=\log -i 2^{k+1} \pi$.

b) Let $\alpha>0$ and $k \geq 0$. Then there is some $a \in \mathbb{C}^{*}$ with $|a|=1$ such that $\overline{z^{\alpha} \circ \tau_{k}}=a z^{\alpha}$.

Proof:

a) Let $z=(r, \varphi) \in T_{k+1}^{\prime}, k \geq 0$. Then by Definition 2.5

$$
\begin{aligned}
\overline{\left(\log \circ \tau_{k}\right)(r, \varphi)} & =\overline{\log \left(r,-\varphi+2^{k+1} \pi\right)} \\
& =\log (r, \varphi)-i 2^{k+1} \pi .
\end{aligned}
$$

b) Let $z=(r, \varphi) \in T_{k+1}^{\prime}, k \geq 0$. Then by Definition 2.5

$$
\begin{aligned}
\overline{\left(z^{\alpha} \circ \tau_{k}\right)(r, \varphi)} & =\overline{\exp \left(\alpha\left(\log r+i\left(-\varphi+2^{k+1} \pi\right)\right)\right.} \\
& =\exp \left(-i \alpha 2^{k+1} \pi\right) z^{\alpha}(r, \varphi) .
\end{aligned}
$$

\section{Lemma 2.16.}

Let $r>0$ and let $f: B(0, r) \rightarrow \mathbb{C}$ be holomorphic and injective with $f(0)=0$ and $\left|f^{\prime}(0)\right|=1$. Then the following holds:

a) $f(B(0, r)) \supset B\left(0, \frac{r}{4}\right)$,

b) $|f(z)| \leq 4|z|$ for $z \in B\left(0, \frac{r}{2}\right)$.

Proof:

a) is a consequence of Koebe's $\frac{1}{4}$-Theorem (see [14, Theorem 2.3]) and b) is a consequence of the Growth Theorem (see [14, Theorem 2.6]).

\section{Theorem 2.17.}

Let $D$ be a simply connected domain with an analytic corner. Let $\Phi: \mathbb{H} \rightarrow D$ be a Riemann map with $\Phi(0)=0$. Then there is a quadratic domain $U$ such that $\Phi$ has a holomorphic extension to $U$.

Proof:

Let $\varphi_{(1)}, \varphi_{(2)}$ and $\Gamma_{1}, \Gamma_{2}$ be as in Definition 1.5. We may assume that the positive direction is mapped to $\Gamma_{1}$ and the negative direction to $\Gamma_{2}$. Replacing $\varphi_{(i)}(z)$ by $\varphi_{(i)}\left(\frac{z}{\left|\varphi_{(i)}^{\prime}(0)\right|}\right)$ we get the following.

There is some $0<\bar{r} \leq 1$ such that $\varphi_{(i)}: B(0, \bar{r}) \rightarrow \mathbb{C}$ is injective, $\left|\varphi_{(i)}^{\prime}(0)\right|=1$ and $\partial D \cap$ $B\left(0, \frac{\bar{r}}{4}\right)=\left(\Gamma_{1} \cup \Gamma_{2}\right) \cap B\left(0, \frac{\bar{r}}{4}\right)$.

Given $k \geq 0$ we recursively define positive real numbers $r_{k}$ and injective holomorphic functions $\varphi_{k} \in \mathcal{O}\left(B\left(0, r_{k}\right)\right), \chi_{k} \in \mathcal{O}\left(B\left(0, \frac{r_{k}}{8}\right)\right)$ with $\varphi_{k}(0)=\chi_{k}(0)=0$ and $\left|\varphi_{k}^{\prime}(0)\right|=\left|\chi_{k}^{\prime}(0)\right|=1$ as follows:

$k=0$ : We choose $0<r_{0} \leq \bar{r}$. We will specify $r_{0}$ below. We take $\varphi_{0}:=\varphi_{(2)} \in \mathcal{O}\left(B\left(0, r_{0}\right)\right)$ and

$$
\chi_{0}: B\left(0, \frac{r_{0}}{8}\right) \longrightarrow \mathbb{C}, z \longmapsto \overline{\varphi_{0}\left(\overline{\varphi_{0}^{-1}(z)}\right)} .
$$

Note that $\chi_{0}$ is well defined: by Lemma 2.16 a) we know that $\varphi_{0}^{-1} \in \mathcal{O}\left(B\left(0, \frac{r_{0}}{4}\right)\right)$. By Lemma $2.16 \mathrm{~b})$ we see that $\left|\varphi_{0}^{-1}(z)\right|<r_{0}$ for $|z|<\frac{r_{0}}{8}$. 
$k \rightarrow k+1$ : We take $r_{k+1}:=\frac{r_{k}}{32}$ and $\varphi_{k+1}: B\left(0, r_{k+1}\right) \rightarrow \mathbb{C}, z \mapsto \overline{\chi_{k}\left(\varphi_{(1)}(\bar{z})\right)}$. We set

$$
\chi_{k+1}: B\left(0, \frac{r_{k+1}}{8}\right) \rightarrow \mathbb{C}, z \mapsto \overline{\varphi_{k+1}\left(\overline{\varphi_{k+1}^{-1}(z)}\right)} .
$$

Note that $\varphi_{k+1}$ is well defined by Lemma $2.16 \mathrm{~b}$ ) and that $\chi_{k+1}$ is well defined by the same argument as in the case $k=0$.

By Fact 2.4 and the subsequent remark we find some $\bar{t}>0$ and some $E>1$ such that $\Phi \in \mathcal{O}(\overline{\mathbb{H}} \cap B(0, \bar{t}))$ and $|\Phi(z)| \leq E|z|^{\alpha}$ for $z \in \overline{\mathbb{H}} \cap B(0, \bar{t})$ where $\alpha:=\varangle D / \pi$.

For $k \geq 0$ we recursively define positive real numbers $t_{k}$ and holomorphic functions $\Phi_{k} \in \mathcal{O}\left(\stackrel{\circ}{T}_{k}\right.$ $\left.\cap B_{\mathbf{L}}\left(t_{k}\right)\right) \cap C^{0}\left(T_{k} \cap B_{\mathbf{L}}\left(t_{k}\right)\right)$ with $\Phi_{k}(0)=0$ and $\Phi_{k}\left(T_{k} \cap B_{\mathbf{L}}\left(t_{k}\right)\right) \subset B\left(0, \frac{r_{k}}{16}\right)$ as follows:

$k=0$ : We choose $0<t_{0} \leq \bar{t}$ such that $\Phi\left(\overline{\mathbb{H}} \cap B\left(0, t_{0}\right)\right) \subset B\left(0, \frac{r_{0}}{16}\right)$. We set $\Phi_{0}:=\Phi$.

$k \rightarrow k+1:$ We define

$$
\Phi_{k+1}: T_{k+1} \cap B_{\mathbf{L}}\left(t_{k}\right) \longrightarrow \mathbb{C}, z \longmapsto \begin{cases}\Phi_{k}(z) & z \in T_{k}, \\ \frac{\chi_{k}\left(\Phi_{k}\left(\tau_{k}(z)\right)\right)}{\text { if }} & z \in T_{k+1}^{\prime} .\end{cases}
$$

Note that $\Phi_{k+1}$ is well defined by the construction of $\chi_{k}$ and the choice of $t_{k}$. Moreover, $\Phi_{k+1}$ is a holomorphic extension of $\Phi_{k}$. We choose $0<t_{k+1} \leq t_{k}$ such that $\Phi_{k+1}\left(T_{k+1} \cap B_{\mathbf{L}}\left(t_{k+1}\right)\right) \subset$ $B\left(0, \frac{r_{k+1}}{16}\right)$. Note that this is possible since $\lim _{|z| \rightarrow 0} \Phi_{k+1}(z)=0$.

So far the only condition imposed on $r_{0}$ is that $0<r_{0}<\bar{r}$. We choose now $r_{0}$ so small such that $\left(\frac{r_{0}}{E \cdot 16}\right)^{\frac{1}{\alpha}} \leq \bar{t}$. For $k \geq 0$ we set $E_{k}:=E 4^{k}$. By induction on $k \geq 0$ we show that we can choose $t_{k}:=\left(\frac{r_{k}}{E_{k} \cdot 16}\right)^{\frac{1}{\alpha}}$ and that $\left|\Phi_{k}(z)\right| \leq E_{k}|z|^{\alpha}$ for $z \in T_{k} \cap B_{\mathbf{L}}\left(t_{k}\right)$.

$k=0$ : By the choice of $r_{0}$ we have $0<t_{0} \leq \bar{t}$. Moreover, $\left|\Phi_{0}(z)\right| \leq E_{0}|z|^{\alpha}$ for $z \in \overline{\mathbb{H}} \cap B_{\mathbf{L}}\left(\overline{t_{0}}\right)$ by the setting. By the definition of $t_{0}$ and $E_{0}$ we see that $\left|\Phi_{0}(z)\right| \leq \frac{r_{0}}{16}$ for $z \in \overline{\mathbb{H}} \cap B_{\mathbf{L}}\left(t_{0}\right)$.

$k \rightarrow k+1$ : We obtain, by applying Lemma $2.17 \mathrm{~b})$ to $\chi_{k} \in \mathcal{O}\left(B\left(0, \frac{r_{k}}{8}\right)\right)$ and by the fact that from the inductive hypothesis we have $\Phi_{k}\left(T_{k} \cap B_{\mathbf{L}}\left(t_{k}\right)\right) \subset B\left(0, \frac{r_{k}}{16}\right)$, that for $z \in T_{k+1}^{\prime} \cap B_{\mathbf{L}}\left(t_{k}\right)$.

$$
\left|\Phi_{k+1}(z)\right|=\left|\chi_{k}\left(\Phi_{k}\left(\tau_{k}(z)\right)\right)\right| \leq 4\left|\Phi_{k}\left(\tau_{k}(z)\right)\right| \leq 4 E_{k}|z|^{\alpha}=E_{k+1}|z|^{\alpha} .
$$

By the definition of $t_{k+1}$ and $E_{k+1}$ we obtain that $\left|\Phi_{k+1}(z)\right| \leq \frac{r_{k+1}}{16}$ for $z \in T_{k+1}^{\prime} \cap B_{\mathbf{L}}\left(t_{k+1}\right)$. Since $\Phi_{k+1}$ coincides with $\Phi_{k}$ on $T_{k}$ the claim follows from the inductive hypothesis.

By construction $\Phi_{k+1}$ extends $\Phi_{k}$ holomorphically for all $k \geq 0$. Hence $\Phi$ has a holomorphic extension to $\bigcup_{k \geq 0} T_{k} \cap B_{\mathbf{L}}\left(t_{k}\right)$. By the definition of $r_{k}$ and $E_{k}$ we obtain some $K>1$ such that $t_{k} \geq K^{-k}$ for all $k \geq 1$. For $\varphi>0$ let $k(\varphi) \in \mathbb{N}$ such that $2^{k(\varphi)-1} \pi \leq \varphi \leq 2^{k(\varphi)} \pi$, i.e. $\mathbb{R}_{>0} \times\{\varphi\} \subset T_{k(\varphi)}^{\prime}$. Then there is some $C>0$ such that $k(\varphi) \leq C \log \varphi$ for all $\varphi>0$ large enough. Enlarging $K>1$, if necessary, we get that $\left\{(r, \varphi) \in \mathbf{L} \mid \varphi>0\right.$ and $\left.r \leq K^{-\log \varphi}\right\} \subset$ $\bigcup_{k \geq 0} T_{k} \cap B_{\mathbf{L}}\left(t_{k}\right)$. Repeating the reflection process in the negative direction we see that $\Phi$ has a holomorphic extension to some quadratic domain $U$ since $\log \varphi \leq \sqrt{\varphi}$ for $\varphi>0$. 


\section{Theorem 2.18.}

Let $D$ be a simply connected domain with an analytic corner. Let $\Phi: \mathbb{H} \rightarrow D$ be a Riemann map with $\Phi(0)=0$. Then there is a quadratic domain $U \subset \mathbf{L}$ such that $\Phi \in \mathcal{Q}^{\log }(U)$. If $\varangle D / \pi \in \mathbb{R} \backslash \mathbb{Q}$ then $\Phi \in \mathcal{Q}(U)$.

Proof:

We use the results and the notation of the previous proof. We define $s_{k}:=\min \left\{t_{k}, E_{k}^{-\frac{2}{\alpha}}\right\}$. Then $\Phi_{k} \in \mathcal{O}\left(\stackrel{\circ}{T}_{k} \cap B_{\mathbf{L}}\left(s_{k}\right)\right) \cap C^{0}\left(T_{k} \cap B_{\mathbf{L}}\left(s_{k}\right)\right)$ and for $z \in T_{k} \cap B_{\mathbf{L}}\left(s_{k}\right)$ we have

$$
\left|\Phi_{k}(z)\right| \leq E_{k}|z|^{\alpha} \leq E_{k}\left|s_{k}^{\frac{\alpha}{2}}\right|\left|z^{\frac{\alpha}{2}}\right| \leq|z|^{\frac{\alpha}{2}} .
$$

Let $g=\sum_{\gamma>0} a_{\gamma} P_{\gamma}(\log z) z^{\gamma} \in \mathbb{C}\left[\left[z^{*}\right]\right]_{\log }$ with $\Phi \sim_{\mathbb{H}} g$ (see Fact 2.4). Given $R>0$ we show that $\Phi(z)-\sum_{\gamma \leq R} a_{\gamma} P_{\gamma}(\log z) z^{\gamma}=o\left(|z|^{R}\right)$ as $|z| \rightarrow 0$ on some quadratic domain $U_{R} \subset U$ which completes the proof. We set

$$
\varepsilon_{R}: \overline{\mathbb{H}} \cap B\left(0, s_{0}\right) \rightarrow \mathbb{C}, z \mapsto \Phi(z)-\sum_{\gamma \leq R} a_{\gamma} P_{\gamma}(\log z) z^{\gamma} .
$$

We choose a constant $S_{R}$ with $R<S_{R}<\min \{\gamma \in \operatorname{supp}(g) \mid \gamma>R\}$. Then by Fact 2.4 and the subsequent remark there is some $C_{R}>1$ such that $\left|\varepsilon_{R}(z)\right| \leq C_{R}|z|^{S_{R}}$ on $\overline{\mathbb{H}} \cap B\left(0, s_{0}\right)$. Shrinking $S_{R}$ and $s_{0}$ (by shrinking the above $r_{0}$ ) we can assume that

$$
\left|\varepsilon_{R}(z)\right| \leq|z|^{S_{R}} \quad \text { on } \quad \overline{\mathbb{H}} \cap B\left(0, s_{0}\right) .
$$

For $k \geq 0$ we set

$$
\varepsilon_{R, k}: T_{k} \cap B_{\mathbf{L}}\left(s_{k}\right) \rightarrow \mathbb{C}, z \mapsto \Phi_{k}(z)-\sum_{\gamma \leq R} a_{\gamma} P_{\gamma}(\log z) z^{\gamma} .
$$

We fix $R>0$ and omit this subscript when it is clear from the context.

Let $\sum_{\ell=1}^{\infty} c_{k, \ell} z^{\ell}$ be the power series expansion of $\chi_{k}$ on $B\left(0, \frac{r_{k}}{8}\right), k \geq 0$. Applying Lemma $\left.2.16 \mathrm{~b}\right)$ and using Cauchy's estimate (see for example [5, IV. 2.14]) we obtain that

$$
\left|c_{k, \ell}\right| \leq 4\left(\frac{16}{r_{k}}\right)^{\ell-1} \quad \text { for } \quad k \geq 0 \text { and } \ell \geq 1
$$

Let $m \in \mathbb{N}$ with $m \alpha / 2>R$ (where $\alpha=\varangle D / \pi$ ). We define

$$
h_{k}: B\left(0, \frac{r_{k}}{8}\right) \longrightarrow \mathbb{C}, z \mapsto \chi_{k}(z)-\sum_{\ell=1}^{m} c_{k, \ell} z^{\ell} .
$$

Claim 1: If $z \in B\left(0, \frac{r_{k}}{16}\right)$ then

$$
\left|h_{k}(z)\right| \leq 4(m+1)\left(\frac{16}{r_{k}}\right)^{m}|z|^{m+1} .
$$

Proof of Claim $\underset{m}{1:}$

By (3) we get $\left|\sum_{\ell=1}^{m} c_{k, \ell} z^{\ell-1}\right| \leq 4 m$ on $B\left(0, \frac{r_{k}}{16}\right)$. Applying the maximum principle to $\sum_{\ell=1}^{m} c_{k, \ell} z^{\ell-1}$ on $B\left(0, \frac{r_{k}}{16}\right)$ we see that $\left|\sum_{\ell=1}^{m} c_{k, \ell} z^{\ell}\right| \leq 4 m|z|$ on $B\left(0, \frac{r_{k}}{16}\right)$ and as a consequence we obtain, again with Lemma $2.16 \mathrm{~b})$ applied to $\chi_{k}$, that $\left|h_{k}(z)\right| \leq 4(m+1)|z|$ on $B\left(0, \frac{r_{k}}{16}\right)$. Again applying the maximum principle to $\frac{h_{k}}{z^{m+1}}$ on $B\left(0, \frac{r_{k}}{16}\right)$ we get Claim 1 . 
We introduce several auxiliary functions in (i), (ii) and (iii) below.

(i) For $k \geq 0$ we define $A_{k} \in C^{0}\left(T_{k+1}^{\prime}\right)$ by $A_{k}(z):=\sum_{\gamma<R} a_{\gamma} P_{\gamma}\left(\log \left(\tau_{k}(z)\right)\right)\left(\tau_{k}(z)\right)^{\gamma}$.

Given $k \geq 1$ we see by Lemma 2.15 that $u_{k}:=\overline{\sum_{\ell=1} c_{k, \ell} A_{k}^{\ell}} \in \mathbb{C}\left[\left[z^{*}\right]\right]_{\log }^{\text {fin }}$. Note that each element of $\operatorname{supp}\left(u_{k}\right)$ is a linear combination of the elements of $\operatorname{supp}(g)$ with positive integers as coefficients. We write $u_{k}=\sum_{\gamma>0} b_{k, \gamma} P_{k, \gamma}(\log z) z^{\gamma} \in \mathbb{C}\left[\left[z^{*}\right]\right]_{\log }^{\mathrm{fin}}$. We set

$$
v_{k}:=\sum_{\gamma \leq R} b_{k, \gamma} P_{k, \gamma}(\log z) z^{\gamma}, w_{k}:=\sum_{\gamma>R} b_{k, \gamma} P_{k, \gamma}(\log z) z^{\gamma} .
$$

Using estimate (3) for the coefficients $c_{k, \ell}$ and Lemma 2.15 for the logarithmic terms we find some $\widehat{L}>1$ independent from $k$ such that after shrinking $S=S_{R}>R$ if necessary

$$
\left|w_{k}(z)\right| \leq \widehat{L}^{k}|z|^{S} \quad \text { for } \quad z \in B_{\mathbf{L}}\left(s_{k}\right) \quad \text { and all } \quad k \geq 0 .
$$

(ii) For $k \geq 0$ we define $Q_{k}(x, y) \in \mathbb{C}[x, y]$ by

$$
Q_{k}(x, y)=\sum_{\ell=1}^{m} c_{k, \ell}\left(\sum_{j=1}^{\ell}\left(\begin{array}{l}
\ell \\
j
\end{array}\right) x^{j} y^{\ell-j}\right)=\sum_{\ell=1}^{m} c_{k, \ell}\left((x+y)^{\ell}-x^{\ell}\right) .
$$

(iii) For $k \geq 0$ we define functions $\Omega_{k}$ on $T_{k} \cap B_{\mathbf{L}}\left(s_{k}\right)$ as follows:

$k=0$ : We set $\Omega_{0} \equiv 0$.

$k \rightarrow k+1$ : We define

$$
\Omega_{k+1}: T_{k+1} \cap B_{\mathbf{L}}\left(s_{k+1}\right), z \longmapsto\left\{\begin{array}{lll}
\Omega_{k}(z) & z \in T_{k}, \\
Q_{k}\left(\varepsilon_{k}\left(\tau_{k}(z)\right), A_{k}(z)\right) & & z \in T_{k+1}^{\prime} .
\end{array}\right.
$$

Claim 2: For every $k \geq 0$

$$
\varepsilon_{k}(z)=o\left(|z|^{R}\right) \quad \text { as } \quad z \mapsto 0 \quad \text { on } \quad T_{k} \cap B_{\mathbf{L}}\left(s_{k}\right)
$$

and

$\left(*_{k}\right) \quad\left|\varepsilon_{k}(z)\right| \leq\left|\Omega_{k}(z)\right|+4(m+1)\left(\frac{16}{r_{k}}\right)^{m}|z|^{\frac{\alpha}{2}(m+1)}+\widehat{L}^{k}|z|^{S} \quad$ for $\quad z \in T_{k} \cap B_{\mathbf{L}}\left(s_{k}\right)$.

We prove Claim 2 by induction on $k$.

$k=0$ : The base case is obvious by (2) and the definition of $\Omega_{0}$.

$k \rightarrow k+1$ : Since $\Phi_{k+1}$ extends $\Phi_{k}$ and since $\Omega_{k+1}$ extends $\Omega_{k}$ the claim holds for $z \in T_{k} \cap$ $B_{\mathbf{L}}\left(s_{k+1}\right)$. Let $z \in T_{k+1}^{\prime} \cap B_{\mathbf{L}}\left(s_{k+1}\right)$. Then

$$
\begin{aligned}
& \Phi_{k+1}(z)=\overline{\chi_{k}\left(\Phi_{k}\left(\tau_{k}(z)\right)\right)} \\
& =\overline{\sum_{\ell=1}^{m} c_{k, \ell}\left(A_{k}(z)+\varepsilon_{k}\left(\tau_{k}(z)\right)\right)^{\ell}+h_{k}\left(\Phi_{k}\left(\tau_{k}(z)\right)\right)} \\
& =\overline{\sum_{\ell=1}^{m} c_{k, \ell}\left(A_{k}(z)\right)^{\ell}}+\overline{Q_{k}\left(\varepsilon_{k}\left(\tau_{k}(z)\right), A_{k}(z)\right)}+\overline{h_{k}\left(\Phi_{k}\left(\tau_{k}(z)\right)\right)} \\
& =v_{k}(z)+w_{k}(z)+\overline{\Omega_{k+1}(z)}+\overline{h_{k}\left(\Phi_{k}\left(\tau_{k}(z)\right)\right)} .
\end{aligned}
$$


THE RIEMANN MAPPING THEOREM FOR SEMIANALYTIC DOMAINS AND O-MINIMALITY

To show $\left(*_{k+1}\right)$ and $\left(* *_{k+1}\right)$ we prove the following

Claim 3: Assuming that $(*)_{k}$ holds we get that $\Phi_{k+1}(z)-v_{k}(z)=o\left(|z|^{R}\right)$ as $z \mapsto 0$ on $T_{k+1}^{\prime} \cap B_{\mathbf{L}}\left(s_{k+1}\right)$.

Proof of Claim 3:

Since $S>R$ we get that $w_{k}(z)=o\left(|z|^{R}\right)$ by (5). Since $m \frac{\alpha}{2}>R$ we get that $h_{k}\left(\Phi_{k}\left(\tau_{k}(z)\right)\right)=$ $o\left(|z|^{R}\right)$ by (1) and (4). Applying $\left(*_{k}\right)$ to $\varepsilon_{k}$ we deduce by the definition of $Q_{k}$ and $A_{k}$ (see also the remarks following Fact 2.4) that $\Omega_{k+1}(z)=o\left(|z|^{R}\right)$. This proves Claim 3 by (6).

We continue with the induction step of Claim 2. For $z \in T_{k} \cap T_{k+1}^{\prime}$ we have $z=\tau_{k}(z)$ and $\Phi_{k+1}(z)=\Phi_{k}(z)$. Therefore we conclude by applying Claim 3, using the inductive hypothesis $\left(*_{k}\right)$, and by the uniqueness of the asymptotic expansion that $v_{k}=\sum_{\gamma<R} a_{\gamma} P_{\gamma}(\log z) z^{\gamma}$. Hence $\varepsilon_{k+1}(z)=\Phi_{k+1}(z)-v_{k}(z)=w_{k}(z)+\overline{\Omega_{k+1}(z)}+\overline{h_{k}\left(\Phi_{k}\left(\tau_{k}(z)\right)\right)}$ for $z \in T_{k+1}^{\prime} \cap B_{\mathbf{L}}\left(s_{k+1}\right)$. The second equality holds by $(6)$. The first equality and Claim 3 give $\left(*_{k+1}\right)$. We obtain $\left(*_{k+1}\right)$ by the second equality and by (1), (4) and (5). So Claim 2 is proven.

By the definition of $r_{k}$ and by $\left(* *_{k}\right)$ we find some $L>1$ independent from $k$ such that

$$
\left|\varepsilon_{k}(z)\right| \leq\left|\Omega_{k}(z)\right|+L^{k}|z|^{T} \quad \text { for } \quad z \in T_{k} \cap B_{\mathbf{L}}\left(s_{k}\right)
$$

where $T:=\min \left\{\frac{\alpha}{2}(m+1), S\right\}$. Note that $T>R$. Using estimate (3) for the coefficients $c_{k, \ell}$ and Lemma 2.15 for the logarithmic terms we can enlarge $L$ (independently from $k$ ) such that for all $k \geq 1$ and $z \in T_{k}^{\prime} \cap B_{\mathbf{L}}\left(s_{k}\right)$

$$
\begin{aligned}
& \left|\Omega_{k}(z)\right|=\left|Q_{k-1}\left(\varepsilon_{k-1}\left(\tau_{k-1}(z)\right), \sum_{\gamma \leq R} a_{\gamma} P_{\gamma}\left(\log \left(\tau_{k-1}(z)\right)\right)\left(\tau_{k-1}(z)\right)^{\gamma}\right)\right| \\
& \leq L^{k}\left(\left|\varepsilon_{k-1}\left(\tau_{k-1}(z)\right)\right|+\left|\varepsilon_{k-1}\left(\tau_{k-1}(z)\right)\right|^{m}\right) .
\end{aligned}
$$

We define $D_{0}:=L$ and recursively $D_{k+1}:=3 L^{k} D_{k}$. By induction on $k \geq 0$ we show that $\left|\varepsilon_{k}(z)\right| \leq D_{k}|z|^{T}$ on $T_{k} \cap B_{\mathbf{L}}\left(p_{k}\right)$ where $p_{0}:=s_{0}$ and $p_{k}:=\min \left\{s_{k}, D_{k-1}^{-T}\right\}$ for $k \geq 1$.

$k=0$ : The base case is a consequence of $(2)$.

$k \rightarrow k+1$ : By the inductive hypothesis we have $\left|\varepsilon_{k}\left(\tau_{k}(z)\right)\right| \leq D_{k}|z|^{T}$ on $T_{k+1}^{\prime} \cap B_{\mathbf{L}}\left(p_{k}\right)$. By the definition of $p_{k}$ and $D_{k}$ we see that $\left|\varepsilon_{k}\left(\tau_{k}(z)\right)\right| \leq 1$ on $T_{k+1}^{\prime} \cap B_{\mathbf{L}}\left(p_{k}\right)$. Using this and (8) we obtain that $\left|\Omega_{k+1}(z)\right| \leq 2 L^{k} D_{k}|z|^{T}$. With (7) we obtain $\left|\varepsilon_{k+1}(z)\right| \leq 3 L^{k} D_{k}|z|^{T}$ and are done.

By the definition of $D_{k}$ and $p_{k}$ we find some $M>1$ such that $\left|\varepsilon_{k}(z)\right| \leq M^{k^{2}}|z|^{T}$ on $T_{k} \cap B_{\mathbf{L}}\left(q_{k}\right)$ where $q_{k}:=M^{-k^{2}} \leq p_{k}, k \geq 0$. We choose $R<\bar{T}<T$ with $T-\bar{T} \leq 1$. We set $\bar{q}_{k}:=M^{-\frac{k^{2}}{T-\bar{T}}}$ and obtain on $T_{k} \cap B_{\mathbf{L}}\left(\bar{q}_{k}\right)$

$$
\left|\Phi_{k}(z)-\sum_{\gamma \leq R} a_{\gamma} P_{\gamma}(\log z) z^{\gamma}\right|=\left|\varepsilon_{R, k}(z)\right| \leq M^{k^{2}}|z|^{T-\bar{T}}|z|^{\bar{T}} \leq|z|^{\bar{T}} .
$$

Using a similar argument as at the end of the proof of Theorem 2.17 we find some $\bar{M}>1$ such that

$$
\left|\Phi(z)-\sum_{\gamma \leq R} a_{\gamma} P_{\gamma}(\log z) z^{\gamma}\right| \leq|z|^{\bar{T}}
$$


on the set $\left\{(r, \varphi) \in \mathbf{L} \mid \varphi>0\right.$ and $\left.r<\bar{M}^{-\left(\log _{+} \varphi\right)^{2}}\right\}$ where $\log _{+} \varphi:=\max \{1, \log \varphi\}$. Repeating the reflection process in the negative direction we see that

$$
\Phi(z)-\sum_{\gamma \leq R} a_{\gamma} P_{\gamma}(\log z) z^{\gamma}=o\left(|z|^{R}\right) \quad \text { as } \quad z \rightarrow 0
$$

on some admissible domain $U_{R} \subset U$ since $\bar{T}>R$ and $(\log \varphi)^{2} \leq \sqrt{\varphi}$ eventually.

So we have proven that $\Phi \in Q^{\log }$. If $\varangle D / \pi \in \mathbb{R} \backslash \mathbb{Q}$ then the asymptotic expansion $g$ of $\Phi$ is an element of $\mathbb{C}\left[\left[z^{*}\right]\right]$ by Fact 2.4. The proof shows that $T \Phi=g$ and therefore $\Phi \in \mathcal{Q}$.

\section{Remark 2.19.}

The proof of the theorem of Lehman (see Fact 2.4 and the subsequent remark) and the proofs of the preceeding Theorems 2.17 and 2.18 show actually the following: let $D$ be a domain with an analytic corner, let $r>0$ and let $\Phi: \mathbb{H} \cap B(0, r) \rightarrow D$ be a holomorphic map with the following properties:

a) $\Phi$ has a continuous extension to $\overline{\mathbb{H}} \cap B(0, r)$ with $\Phi(0)=0$.

b) $\Phi\left(\left[0, r[) \subset \Gamma_{1}\right.\right.$ and $\left.\left.\Phi(]-r, 0\right]\right) \subset \Gamma_{2}$.

Then there is a quadratic domain $U \subset \mathbf{L}$ such that $\Phi \in \mathcal{Q}^{\log }(U)$. If $\varangle D / \pi \in \mathbb{R} \backslash \mathbb{Q}$ then $\Phi \in \mathcal{Q}(U)$.

\section{Proof of Theorem 2.12:}

The Riemann map $\Phi: \mathbb{H} \rightarrow \Omega$ maps 0 to a boundary point $x \in \partial \Omega$ with attached angle $\varangle$ greater than 0 . After some translation of the domain $\Omega$ we can assume that $x=0$. Let $C$ be a simply connected and semianalytic domain such that $C$ is representative of the germ of $\Omega$ at 0 to which the germ of $\mathbb{H}$ at 0 is mapped by $\Phi$. We choose $C$ such that $\partial C \cap \partial \Omega$ consists of two semianalytic branches $\Gamma_{1}$ and $\Gamma_{2}$ with $\Gamma_{1} \cap \Gamma_{2}=\{0\}$. Note that $\varangle_{0} C=\varangle$. Moreover, we choose $C$ in such a way that after some rotation $\rho$ there is a convergent Puiseux series $\psi_{1}:\left[0, \delta\left[\rightarrow \mathbb{R}\right.\right.$ with $\Gamma_{1}^{*}=\left\{\left(t, \psi_{1}(t)\right) \mid 0 \leq t<\delta\right\}$ where $\Gamma_{1}^{*}:=\rho\left(\Gamma_{1}\right)$. This can be done by analytic cell decomposition (see [10, pp.508-509]) and the fact that subanalytic functions in one variable are given by such series (see for example $[7, \mathrm{p} .192]$ ). There is some $d \in \mathbb{N}$ and some convergent real power series $\left.\chi_{1}:\right]-\delta^{d}, \delta^{d}\left[\rightarrow \mathbb{R}\right.$ such that $\psi_{1}(t)=\chi_{1}\left(t^{\frac{1}{d}}\right), t \geq 0$. Hence $\Gamma_{1}^{*}=\left\{\left(t^{d}, \chi_{1}(t)\right) \mid 0 \leq t<\delta^{d}\right\}$. We consider $\varphi_{1}^{*}: B\left(0, \delta^{d}\right) \rightarrow \mathbb{C}, z \mapsto z^{d}+i \chi_{1}(z)$. Then $\varphi_{1}^{*} \in \mathcal{O}\left(B\left(0, \delta^{d}\right)\right)$ and $\Gamma_{1}^{*}=\varphi_{1}^{*}\left(\left[0, \delta^{d}[)\right.\right.$. By the same argument applied to $\Gamma_{2}$ we find (after back-rotation and some dilatation) holomorphic functions $\varphi_{1}, \varphi_{2} \in \mathcal{O}(B(0,1))$ with $\varphi_{1}(0)=\varphi_{2}(0)=0$ such that $\Gamma_{1}=\varphi_{1}\left(\left[0,1[)\right.\right.$ and $\Gamma_{2}=\varphi_{2}\left(\left[0,1[)\right.\right.$. Let $m_{1}, m_{2} \in \mathbb{N}$ be such that $\varphi_{1}(z)=z^{m_{1}} \widehat{\varphi}_{1}(z), \varphi_{2}(z)=z^{m_{2}} \widehat{\varphi}_{2}(z)$ where $\widehat{\varphi}_{1}, \widehat{\varphi}_{2} \in \mathcal{O}(B(0,1))$ and $\widehat{\varphi}_{1}(0) \cdot \widehat{\varphi}_{2}(0) \neq 0$. We can replace $\varphi_{2}(z)$ by $\varphi_{2}\left(z^{m_{1}}\right)$ and can therefore assume that $m_{1}$ divides $m_{2}$.

Let $r>0$ such that $\Phi(\overline{\mathbb{H}} \cap B(0, r)) \subset \bar{C}$ and $\Phi\left(\left[0, r[) \subset \Gamma_{1}, \Phi(]-r, 0\right]\right) \subset \Gamma_{2}$ (we switch 1 and 2 if necessary). We apply finitely many elementary transformations to $\Phi$ and $C$. We obtain functions $\Phi^{(i)}$ and domains $C^{(i)}, i=1,2,3$, such that $C^{(3)}$ is a domain with an analytic corner. The domains $C^{(i)}$ allow a similar description as $C$. We denote the corresponding data describing $C^{(i)}$ by $\Gamma_{1,2}^{(i)}, \varphi_{1,2}^{(i)}$ and $m_{1,2}^{(i)}$.

1) We consider $\Phi^{(1)}: \mathbb{H} \cap B(0, r) \rightarrow C^{(1)}, z \mapsto \Phi(z)^{\frac{1}{m_{1}}}$, where $C^{(1)}:=(C)^{\frac{1}{m_{1}}}$ (we take an appropriate $m_{1}$-th root of $\Phi$ and on $C$; note that $C$ is simply connected). Since $m_{1}$ divides 
THE RIEMANN MAPPING THEOREM FOR SEMIANALYTIC DOMAINS AND O-MINIMALITY 19 $m_{2}$ we see that $C^{(1)}$ has a similar description as $C$ but additionally $m_{1}^{(1)}=1$. Moreover, $\varangle_{0} C^{(1)}=\varangle / m_{1}$.

2) We consider (after shrinking $r>0$ if necessary) $\Phi^{(2)}: \mathbb{H} \cap B(0, r) \rightarrow C^{(2)}, z \mapsto-\left(\varphi_{1}^{(1)}\right)^{-1} \Phi^{(1)}(z)$, where $C^{(2)}:=-\left(\varphi_{1}^{(1)}\right)^{-1}\left(C^{(1)}\right)$. Note that $\varphi_{1}^{(1)}$ is invertible at 0 since $m_{1}^{(1)}=1$. We can choose $C$ a priori such that $\left(\varphi_{1}^{(1)}\right)^{-1} \in \mathcal{O}(B(0, s))$ and $C^{(1)} \subset B(0, s)$ for some $s>0$. We have that $\Gamma_{1}^{(2)} \subset \mathbb{R}_{\leq 0}$. Moreover, $\varangle_{0} C^{(2)}=\varangle_{0} C^{(1)}$.

3) We consider $\Phi^{(3)}: \mathbb{H} \cap B(0, r) \rightarrow C^{(3)}, z \mapsto \rho\left(\Phi^{(2)}(z)\right)^{\frac{1}{m_{2}^{(2)}}}$, where $C^{(3)}:=\rho\left(C^{(2)}\right)^{\frac{1}{m_{2}^{(2)}}}$ and $\rho \in \mathbb{C}$ with $|\rho|=1$ such that $\Gamma_{1}^{(3)} \subset \mathbb{R}_{\leq 0}$. Then $m_{2}^{(3)}=1$. Moreover, $\varangle_{0} C^{(3)}=$ $\varangle_{0} C^{(2)} / m_{2}^{(2)}$.

By construction $C^{(3)}$ has an analytic corner. By Theorem 2.18 and the subsequent Remark 2.19 we get that $\Phi^{(3)} \in \mathcal{Q}^{\log }$ and $\Phi^{(3)} \in \mathcal{Q}$ if $\varangle C^{(3)} \in \pi(\mathbb{R} \backslash \mathbb{Q})$. We have $\Phi=\left(\varphi_{1}^{(1)}\left(-\left(\rho^{-1} \Phi^{(3)}\right)^{m_{2}^{(2)}}\right)\right)^{m_{1}}$. Generalizing [21, Proposition 3.9] to $\mathcal{Q}^{\log }$ and using the fact that $\mathcal{Q}^{\log }$ (resp. $\mathcal{Q}$ ) is a $\mathbb{C}$-algebra (see Remark 2.10) we get that $\Phi \in \mathcal{Q}^{\log }$ and that $\Phi \in \mathcal{Q}$ if $\Phi^{(3)} \in \mathcal{Q}$. We have $\Phi^{(3)} \in \mathcal{Q}$ if $\varangle_{0} C^{(3)} / \pi \in \mathbb{R} \backslash \mathbb{Q}$ and the latter is the case iff $\varangle_{0} C / \pi \in \mathbb{R} \backslash \mathbb{Q}$.

\section{Riemann maps at semianalytic domains and o-minimality}

Now we are able to prove Theorem A. As mentioned in the introduction the singular boundary points are the difficult part. We use Theorem 2.12. In [21] it was shown that the functions of $\mathcal{Q}$ restricted to the real line generate an o-minimal structure, denoted by $\mathbb{R}_{\mathcal{Q}}$. We show that the Riemann map is definable in the o-minimal structure $\mathbb{R}_{\mathcal{Q}}$ as a two variable function. We use polar coordinates.

Definition 3.1 (compare with [21, Definition $3.4 \&$ Definition 4.3]).

Let $\lambda \in \overline{\mathbb{H}} \backslash\{0\}$. We have $B(|\lambda|,|\lambda|)) \subset \mathbf{L}$ via the identification of $\mathbb{C} \backslash \mathbb{R}_{\leq 0}$ with $\left.\mathbb{R}_{>0} \times\right]-\pi, \pi[\subset \mathbf{L}$. Let $\lambda=|\lambda| e^{i a}$ with $0 \leq a \leq \pi$. We identify $B(\lambda,|\lambda|)$ with $\{(r, \varphi) \in \mathbf{L} \mid(r, \varphi-a) \in B(|\lambda|,|\lambda|)\}$. We set $\mathbf{t}_{\lambda}: B(0,|\lambda|) \rightarrow B(\lambda,|\lambda|), z \mapsto \lambda+z$, and for $\rho>0$ we define $\mathbf{r}^{\rho, \lambda}: \mathbf{L} \times B(0,|\lambda|) \rightarrow \mathbf{L}^{2}$, $\left(z_{1}, z_{2}\right) \mapsto\left(z_{1}, w_{2}\right)$, with $w_{2}:=\mathbf{m}\left(\mathbf{p}^{\rho}\left(z_{1}\right), \mathbf{t}_{\lambda}\left(z_{2}\right)\right)$ (see Definition 2.5 for the definition of $\mathbf{p}$ and $\mathbf{m})$.

\section{Remark 3.2.}

Let $U \subset \mathbf{L}^{2}$ be a 2-quadratic domain (compare with [21, Definition 2.4]) and let $f \in \mathcal{Q}_{2}^{2}(U)$ (compare with [21, Definition 5.1]). Let $\lambda \in \overline{\mathbb{H}} \backslash\{0\}$. As in [21, Proposition 4.4 \& Proposition 5.15] we find some 1-quadratic domain $V \subset \mathbf{L} \times B_{\mathbf{L}}(|\lambda|)$ such that $\mathbf{r}^{1, \lambda}(V) \subset U$ and the function $\mathbf{r}^{1, \lambda} f:=f \circ \mathbf{r}^{1, \lambda} \in \mathcal{Q}_{1}^{2}(V)$.

Theorem A gets the following precise form:

\section{Theorem 3.3.}

Let $\Omega \subset \mathbb{C}$ be a bounded, semianalytic and simply connected domain. Suppose that $\varangle(\Omega, x) \subset$ $\pi(\mathbb{R} \backslash \mathbb{Q})$ for all $x \in \operatorname{Sing}(\partial \Omega)$. Let $F: \Omega \rightarrow B(0,1)$ be a Riemann map. Then $F$ is definable in the o-minimal structure $\mathbb{R}_{\mathcal{Q}}$. 
Proof:

We establish a local definability result: given $x \in \bar{\Omega}$ we show that $\left.F\right|_{B(x, r)}$ is definable in $\mathbb{R}_{\mathcal{Q}}$ for some $r>0$. Then we use compactness of $\bar{\Omega}$ to obtain the theorem.

Let $x \in \bar{\Omega}$.

Case 1: $x \in \Omega$. Let $r:=\operatorname{dist}(x, \partial \Omega)$. Then $\left.F\right|_{B(x, r)}$ is real analytic, hence $\left.F\right|_{B\left(x, \frac{r}{2}\right)}$ is definable in $\mathbb{R}_{\text {an }}$ which is a reduct of $\mathbb{R}_{\mathcal{Q}}$.

Case 2: $x \in \partial \Omega \backslash \operatorname{Sing}(\partial \Omega)$. Then the germ of $\Omega$ at $x$ has one or two components (compare with Example 1.3). Let $C_{*}$ be a semianalytic representative of such a component. By the Schwarz reflection principle there is some $r>0$ such that $F$ has a holomorphic extension to $B(x, r)$. So $\left.F\right|_{C_{*} \cap B\left(x, \frac{r}{2}\right)}$ is definable in $\mathbb{R}_{\mathrm{an}}$.

Case 3: $x \in \operatorname{Sing}(\partial \Omega)$. Let $C$ be a component of the germ of $\Omega$ at $x$ and let $C_{*}$ be a semianalytic and simply connected domain which is a representative of $C$. If $x \notin \operatorname{sing}\left(\partial C_{*}\right)$ we can argue as in Case 2. So we assume that $x \in \operatorname{Sing}\left(\partial C_{*}\right)$. Then $\varangle_{x} C_{*} \in \pi(\mathbb{R} \backslash \mathbb{Q})$ by assumption. Let $j: \mathbb{H} \rightarrow B(0,1)$ be a suitable Möbius transformation such that $\Phi:=F^{-1} \circ j$ maps the germ of $\mathbb{H}$ at 0 to $C$. We show that there is some $r>0$ such that $\left.\Phi\right|_{\mathbb{H} \cap B(0, r)}$ is definable in $\mathbb{R}_{\mathcal{Q}}$. By Theorem 2.12 and the assumption we have some quadratic domain $U \subset \mathbf{L}$ such that $\Phi \in \mathcal{Q}(U)$. We define $f: U \times U \rightarrow \mathbb{C},\left(z_{1}, z_{2}\right) \mapsto \Phi\left(z_{2}\right)$. Let $a \in[0, \pi]$. We consider $g_{a}:=\mathbf{r}^{1, \lambda_{a}} f$ with $\lambda_{a}:=e^{i a}$. By Remark 3.2 we get that $g_{a} \in \mathcal{Q}_{1}^{2}$. We set $G_{a}:=g_{a}\left(z_{1}, h_{a}\left(z_{2}\right)\right)$ with $h_{a}(z):=e^{i(z+a)}-e^{i a}$. Then $G_{a} \in \mathcal{Q}_{1}^{2}$ by [21, Proposition 5.10]. Hence there is some $r_{a}>0$ and some quadratic domain $U_{a}$ such that $G_{a} \in \mathcal{Q}_{1}^{2}\left(U_{a} \times B\left(0, r_{a}\right)\right)$. We can assume that $U_{a}=\left\{(r, \varphi) \in \mathbf{L} \mid 0<r<c_{a} \exp \left(-C_{a} \sqrt{|\varphi|}\right)\right\}$ with some positive constants $c_{a}, C_{a}$. We define $\overline{G_{a}}: U_{a} \times B\left(0, r_{a}\right) \rightarrow \mathbb{C},\left(z_{1}, z_{2}\right) \mapsto \overline{G_{a}\left(\overline{z_{1}}, \overline{z_{2}}\right)}$ where we set $\bar{z}:=(r,-\varphi)$ for $z=(r, \varphi) \in L$. We also denote with $\bar{z}$ the complex conjugate of a complex number $z$. Note that $\overline{G_{a}} \in \mathcal{Q}_{1}^{2}$ (compare with [21, Proposition 7.3]). We set $\mathcal{R} G_{a}:=\frac{1}{2}\left(G_{a}+\overline{G_{a}}\right)$ and $\mathcal{J} G_{a}:=\frac{1}{2 i}\left(G_{a}-\overline{G_{a}}\right)$. Then $\mathcal{R} G_{a}, \mathcal{J} G_{a} \in \mathcal{Q}_{1,1 ; \varepsilon_{a}}$ for some $\varepsilon_{a}>0$ (compare with [21, Section 7]). Hence $\mathcal{R} G_{a}$ and $\mathcal{J} G_{a}$ are defined on $I_{a}:=\left[0, \varepsilon_{a}\right] \times\left[-\varepsilon_{a}, \varepsilon_{a}\right]$, and $\left.\mathcal{R} G_{a}\right|_{I_{a}}$ and $\left.\mathcal{J} G_{a}\right|_{I_{a}}$ are definable in $\mathbb{R}_{\mathcal{Q}}$.

For $(r, \varphi) \in I_{a}$ we get $\mathcal{R} G_{a}(r, \varphi)=\operatorname{Re} \Phi\left(r e^{i(\varphi+a)}\right)=\operatorname{Re} \Phi(r \cos (\varphi+a), r \sin (\varphi+a))$ and $\mathcal{J} G_{a}(r, \varphi)=\operatorname{Im} \Phi\left(r e^{i(\varphi+a)}\right)=\operatorname{Im} \Phi(r \cos (\varphi+a), r \sin (\varphi+a))$. Since the polar coordinates are definable in $\mathbb{R}_{\mathcal{Q}}$ we find by a compactness argument (note that $a \in[0, \pi]$ ) some $r>0$, such that $\left.\Phi\right|_{\mathbb{H} \cap B(0, r)}$ is definable in $\mathbb{R}_{\mathcal{Q}}$. Since the Möbius transformation is semialgebraic we find some $s>0$ such that $\left.F\right|_{C_{*} \cap B(x, r)}$ is definable in $\mathbb{R}_{\mathcal{Q}}$. Doing this argument for the finitely many connected components of the germ of $\Omega$ at $x$ we obtain the claim.

\section{Corollary 3.4.}

Let $\Omega \varsubsetneqq \mathbb{C}$ be a globally semianalytic and simply connected domain. Suppose that $\varangle(\Omega, x) \subset$ $\pi(\mathbb{R} \backslash \mathbb{Q})$ for all $x \in \operatorname{Sing}\left(\partial^{\infty} \Omega\right)$. Let $F: \Omega \rightarrow B(0,1)$ be a Riemann map. Then $F$ is definable in the o-minimal structure $\mathbb{R}_{\mathcal{Q}}$.

Proof:

We can copy the proof of Theorem 3.3. For $x=\infty$ we work with $\Omega^{\prime}$ and 0, see Definition 1.4. 
THE RIEMANN MAPPING THEOREM FOR SEMIANALYTIC DOMAINS AND O-MINIMALITY 21

\section{Corollary 3.5.}

Let $\Omega_{1}, \Omega_{2} \varsubsetneqq \mathbb{C}$ be globally semianalytic and simply connected domains with $\varangle\left(\Omega_{1}, x\right) \subset$ $\pi(\mathbb{R} \backslash \mathbb{Q})$ and $\varangle\left(\Omega_{2}, x\right) \subset \pi(\mathbb{R} \backslash \mathbb{Q})$ for all $x \in \operatorname{Sing}\left(\partial^{\infty} \Omega_{1}\right)$ and all $x \in \operatorname{Sing}\left(\partial^{\infty} \Omega_{2}\right)$. Then each biholomorphic map $\Omega_{1} \rightarrow \Omega_{2}$ is definable in $\mathbb{R}_{\mathcal{Q}}$.

\section{Remark 3.6.}

We consider special cases of domains.

a) If the domain in question is a polygon $P$ a Riemann map $\mathbb{H} \rightarrow P$ is a so-called SchwarzChristoffel map (see for example Fischer-Lieb [16, p.208]). In the case of an rectangle the Schwarz-Christoffel map is given by an elliptic integral.

For polygons we can overcome the restriction on the angles at singular boundary points by [20, Proposition 2]: a Riemann map $\mathbb{H} \rightarrow P$ is definable in the o-minimal structure $\mathbb{R}_{\mathrm{an}}^{\mathbb{R}}$ which is a reduct of $\mathbb{R}_{\mathcal{Q}}$.

b) If the domain in question is a circular polygon $C$, then a Riemann map $\mathbb{H} \rightarrow C$ fulfills the Schwarz differential equation (compare with [16, Theorem VI.4.4]). The solutions to the Schwarz differential equation are exactly quotients of independent solutions to the hypergeometric differential equation (compare with [16, Satz VI.5.3]). Applying Theorem 3.3 we obtain that these functions are definable in $\mathbb{R}_{\mathcal{Q}}$ if $\varangle_{x} C \in \pi(\mathbb{R} \backslash \mathbb{Q})$ for all $x \in$ $\operatorname{Sing}(\partial C)$.

\section{References}

1. G.V. Badalyan, Quasipower Series and Quasianalytic Classes of Functions. Translation of Mathematical Monographs Vol. 216, AMS, 2002.

2. E. Bierstone, P.D. Milman, Semianalytic and subanalytic sets. Inst. Hautes Études Sci. Publ. Math. 67 (1988), pp. 5-42.

3. J. Bochnak, M. Coste, M.-F. Roy, Real Algebraic Geometry. Springer, 1998.

4. G. Comte, J.-M. Lion, J.-P. Rolin, Nature log-analytique du volume des sous-analytiques. Illinois J. Math. 44, no.4 (2000), pp. 884-888.

5. J.B. Conway, Functions of One Complex Variable. Second Edition. Springer, 1978.

6. J. Denef, L. VAn den Dries, p-adic and real subanalytic sets. Annals of Mathematics (2) 128, no.1 (1988), pp. $79-138$.

7. L. VAn Den Dries, A generalization of the Tarski-Seidenberg Theorem, and some nondefinability results. Bull. Am. Math. Soc. 15 (1986), pp. 189-193.

8. L. van den Dries, Tame Topology and O-minimal Structures. London Math. Soc. Lecture Notes Series 248 (1998), Cambridge University Press.

9. L. van den Dries, A. Macintyre, D. Marker, The elementary theory of restricted analytic functions with exponentiation. Annals of Math. (2) 140, no.1 (1994), pp. 183-205.

10. L. van den Dries, C. Miller, Geometric categories and o-minimal structures. Duke Math. J. 84 (1996), pp. $497-540$.

11. L. van den Dries, P. Speissegger, The real field with convergent generalized power series. Trans. Amer. Math. Soc. 350, no.11 (1998), pp. 4377-4421.

12. L. van den Dries, P. Speissegger, The field of reals with multisummable series and the exponential function. Proc. London Math. Soc. (3) 81, no.3 (2000), pp. 513-565.

13. H. DulaC, Sur les cycles limites. Bull. Soc. Math. France 51 (1923), pp. 45-188.

14. P.L. Duren, Univalent Functions. Springer, 1993.

15. G. Efroymson, The Riemann Mapping Theorem for planar Nash rings. Pacific J. Math. 92, no.1 (1981), pp. 73-78.

16. W. Fischer, I. Lieb, Ausgewählte Kapitel der Funktionentheorie. Vieweg, 1988. 
17. R. Huber, M. Knebusch, A glimpse at isoalgebraic spaces. Note Mat. 10, suppl.2 (1990), pp. 315-336.

18. Y.S. Ilyashenko, Finiteness Theorems for Limit Cycles. Translations of Mathemat. Monographs 94, American Mathematical Society, 1991.

19. Y.S. Ilyashenko, Centennial history of Hilbert's 16th problem. Bull. Amer. Math. Soc. 39, no.3 (2002), pp. 301-354.

20. T. KAISER, Definability results for the Poisson equation. Adv. Geom.6 (2006), pp. 627-644.

21. T. Kaiser, J.-P. Rolin, P. Speissegger, Transition maps at non-resonant hyperbolic singularities are o-minimal. To appear at Journal für die reine und angewandte Mathematik. arXiv:math/0612745

22. M. Knebusch, Isoalgebraic geometry: first steps. Seminar on Number Theory, Paris 1980-81, pp. 127-141, Progr. Math. 22, (1982), Birkhäuser.

23. R.S. Lehman, Development of the mapping function at an analytic corner. Pacific J. of Math. 7 (1957), pp. 1437-1449.

24. S. Lojasiewicz, Ensemble Semianalytiques. Institut des Hautes Etudes Scientifiques, Bures-sur-Yvette, France, 1965.

25. C. Miller, Expansions of the real field with power functions. Ann. Pure Appl. Logic 68, no.1 (1994), pp. 79-94.

26. Y. Peterzil, S. Starchenko, Expansions of algebraically closed fields in o-minimal structures. Selecta Math. (N.S) 7, no.3 (2000), pp. 409-445.

27. Y. Peterzil, S. Starchenko, Expansions of algebraically closed fields in o-minimal structures. II. Functions of several variables. J. Math. Logic 3, no.1 (2003), pp. 1-35.

28. Ch. Pommerenke, Boundary Behaviour of Conformal Maps, Springer, 1991.

29. R. Remmert, Classical Topics in Complex Function Theory, Springer, 1997.

30. J.-P. Rolin, P. Speissegger, A.J. Wilkie, Quasianalytic Denjoy-Carleman classes and o-minimality. J. Amer. Math. Soc. 16, no.4 (2003), pp. 751-777.

31. M. SнiотA, Geometry of subanalytic and semialgebraic sets. Birkhäuser, 1997.

32. N.M. Wigley, Development of the mapping function at a corner. Pacific J. of Math. 15 (1965), pp. 14351461.

33. A.J. WiLkie, Model completeness results for expansions of the ordered field of real numbers by restricted Pfaffian functions and the exponential function. Journal Amer. Math. Soc. 9, no.4 (1996), pp. 1051-1094.

Tobias Kaiser

University of Regensburg

Department of Mathematics

Universitätsstr. 31

D-93040 Regensburg

Germany

Tobias.Kaiser@mathematik.uni-regensburg.de 